\title{
SVD analysis of the magnetospheric AE index time series and comparison with low-dimensional chaotic dynamics
}

\author{
M. A. Athanasiu and G. P. Pavlos \\ Department of Electrical and Computer Engineering, Demokritos Univercity of Thrace, 67100 Xanthi, Greece
}

Received: 12 November 1999 - Accepted: 9 May 2000

\begin{abstract}
The singular value decomposition (SVD) analysis is used at different stages in this paper in order to extract useful information concerning the underlying dynamics of the magnetospheric AE index. As a frame of reference we use the dynamics of the Lorenz system perturbed by external noise, white or colored. One of the critical results is that the colored noise can be differentiated from the white noise when we study their perturbation upon the eigenvalue spectrum of the trajectory matrix, the SVD reconstructed components of the original time series and other characteristics. This result is used in order to conclude the existence of strong component of colored noise included in the magnetospheric AE index time series. Moreover, the study of the SVD reconstructed components of the original time series can confirm the low-dimensionality of a dynamical system strongly perturbed by external colored noise. Finally, the results of this study strengthen the hypothesis of the magnetospheric chaos.
\end{abstract}

\section{Introduction}

The generalized theory known as singular system analysis or singular value decomposition (SVD) analysis constitutes a significant tool for the discrimination of different dynamical components included in an experimental time series. SVD analysis was recently developed by Broomhead and King (1986) in relation with chaotic time series analysis as a framework which allows one to address the problems associated with the noisy, finite precision sampled data produced by measuring experimental phenomenon. Broomhead and King (1986) used the SVD analysis in order to exclude the noisy component associated with small or vanishing singular values $\left(\sigma_{i}\right)$ corresponding to the principal axes in the embedding space.

Additionally, in this paper we use SVD analysis in order to exclude "noisy" components associated with large singular values, especially the $\sigma_{1}$ singular value corresponding to the

Correspondence to: M. Athanasiu first principal axis and the $c_{1}$ eigenvector of the SVD analysis, according to the ordering $\sigma_{1} \geq \sigma_{2} \geq \ldots \geq \sigma_{n} \geq 0$ (see the next section). In this direction it has been proved that the SVD analysis strongly supports the low-dimensional character of the internal magnetospheric dynamics as it is revealed in the AE index time series. Ten years ago we used the concept of strange attractor dynamics as an explicative paradigm of the magnetospheric substorms (see Pavlos, 1988). According to this concept it is implied that the magnetospheric substorms can be explained as the effect of nonlinear dynamics of the magnetospheric physical state on a strange attracting subset of the phase-space corresponding to the magnetospheric dynamics. Baker et al. (1990) have studied the solar-wind magnetosphere coupling problem using a nonlinear dripping faucet analogy of the system. This approach was motivated by using the laboratory study of the dripping faucet (Shaw, 1984) and also by using the dripping faucet description of plasmoid formation and release discussed by Hones (1979). Baker's model of the magnetospheric dynamics is a mechanical analog. Klimas et al. (1991, 1992) developed the Faraday loop response model. Pavlos et al. (1994) extended the linear magnetospheric equivalent electric circuit of Liu et al. (1988) to a nonlinear one. The above nonlinear modeling of the magnetospheric dynamics has given strong results supporting the concept of magnetospheric chaos which has also been indicated by chaotic analysis of experimental time series (Vassiliadis et al., 1990; Shan et al., 1991; Roberts et al., 1991; Prichard and Price, 1992; Pavlos et al., 1992a, b; Vassiliadis et al., 1992; Sharma et al., 1993; Takalo and Timonen, 1994).

Parallel to these studies a fruitful criticism has been developed about the hypothesis of the magnetospheric chaos especially in relation to its experimental evidence. Prichard and Price $(1992,1993)$ showed that many of the previous results supporting experimentally the concept of low-dimensional magnetospheric dynamics were caused by the long decorrelation time of the $\mathrm{AE}$ index and therefore were not the result of low-dimensional dynamics. Osborne and Provenzale (1989), Provenzale et al. (1992), Theiler (1991), Pavlos et al. 
(1992a, b) used many tests in order to exclude the pseudochaos of colored noises. The term pseudo-chaos was introduced by Pavlos et al. (1992a, b) to discriminate the real lowdimensional chaotic dynamics contained in aperiodic time series from the stochastic high dimensional time series (nonchaotic and aperiodic time series) which can mimic almost indistinguishably the phenomenology of chaotic time series. Moreover, Vassiliadis et al. (1992) used the Theiler's test in the case of magnetospheric data and showed that, when the parameter $w$ of Theiler becomes comparable to the decorrelation time of the magnetospheric signal, the scaling in the correlation integral disappears and there is no convergence of its slopes. Pavlos et al. (1994) extended the chaotic analysis to the AE index by using SVD analysis according to Broomhead and King (1986). Sharma et al. (1993) had also used SVD analysis for the estimation of the eigenvalue spectrum of the AE index. The combination of SVD analysis and the Theiler's test in the work of Pavlos et al. (1994) has given strong evidence for the existence of magnetospheric chaos especially when the Theiler's parameter $w$ is equal to the decorrelation time. Prichard (1995), in a short comment, strongly criticizes the results presented in the work of Pavlos et al. (1994). In this criticism he uses the method of surrogate data and the method of Takens (1985) for the estimation of correlation dimension. The conclusion by Prichard was that there is no evidence that the AE index can be described by a low-dimensional strange attractor. An extended review of studies of nonlinear dynamics of the magnetosphere is given by Klimas et al. (1996). In two previous studies (Pavlos et al., 1999a, b) we have ascertained that the detailed statistical comparison of geometrical and dynamical magnitudes corresponding to the AE index time series and its nonlinear surrogate data (Theiler et al., 1992a,b) can reveal significant discrimination between the AE index and the non-linear noises (nonlinear surrogate data). These results strongly indicate the non-linearity and low-dimensionality of the AE index. Furthermore, the comparison of the magnetospheric dynamics with known stochastic and input-output dynamics which can mimic the magnetosperic system (Pavlos et al., 1999c) has shown that the low-dimensional chaotic dynamics is the most appropriate concept for modeling the magnetospheric system. In the last paper, it was also shown that the study of the SVD reconstructed components of the AE index can indicate the existence of an external high dimensional dynamical component.

In this paper we extend the previous results and we compare the SVD spectrum of the reconstructed components of the AE index time series with the SVD reconstructed components of the Lorenz system. In Section 2 we summarize the theory of SVD analysis concerning the SVD components of the original time series. In Section 3 we present the results of the SVD analysis applied to the magnetospheric AE index time series. In Section 4 we present results of the SVD analysis applied to the Lorenz system perturbed by external noise. In the same section we compare the results of the SVD analysis applied to both cases, the Lorenz and the magnetospheric systems and we extract significant conclusions. In
Section 5 we study the two-dimensional phase portraits of the SVD reconstructed components of the AE index and the Lorenz system perturbed by external noise. In Section 6 we compare the cross correlation and the standard deviation of the original signals and their SVD reconstructed components in the case of the AE index and the Lorenz system which is also perturbed by external noise. Finally, in Section 7, we summarize the new results of this study especially with the hypothesis of magnetospheric chaos and we raise critical questions for future study.

\section{Theoretical framework}

In this section we summarize some theoretical concepts concerning the embedding theory, the method of surrogate data and the SVD analysis which constitute the main tools of our analysis.

\subsection{Embedding theory and phase-space reconstruction}

The earth's magnetosphere is a system of magnetized plasma, which microscopically is an infinite dimensional system, the dynamics of which is mirrored in the ground measured AE index. Some kind of "self-organization" may give rise to the system evolution on a low-dimensional manifold $\mathbf{M}$ of dimension $d$. This means that the magnetosphere can be described macroscopically by a low-dimensional dynamical system of $n$ macroscopic degrees of freedom with $n \geq d$. For linear systems, "self-organization" is more an externally driven process described by the external parameters of the system. For nonlinear and dissipative systems, however, it is possible that the system evolves by its internal dynamics in such a way that the corresponding phase space flow contracts on sets of lower dimensions which are called attractors.

The embedding theory permits one to study the dynamical characteristics of a physical system by using experimental observations in the form of time series (Takens, 1981; Broomhead and King, 1986). Let $\boldsymbol{x}(t)=f^{(t)}(\boldsymbol{x}(0))$ denote the dynamical flow underlying an experimental time series $x\left(t_{i}\right)=h\left(\boldsymbol{x}\left(t_{i}\right)\right)$ where $h$ describes the measurement function. When there is a noisy component $w\left(t_{i}\right)$ then the observed time series must be given by $x\left(t_{i}\right)=$ $h\left(\boldsymbol{x}\left(t_{i}\right), w\left(t_{i}\right)\right)$. On the other hand, Takens (1981) showed that for autonomous and purely deterministic systems the delay reconstruction map $\Phi$, which maps the states $\boldsymbol{x}$ into mdimensional delay vectors

$\Phi(\boldsymbol{x})=\left[h(\boldsymbol{x}), h\left(f^{\tau}(\boldsymbol{x})\right), h\left(f^{2 \tau}(\boldsymbol{x})\right), \ldots, h\left(f^{(m-1) \tau}(\boldsymbol{x})\right)\right]$

is an embedding when $m \geq 2 n+1$, where $n$ is the dimension of the manifold $\mathbf{M}$ of the phase space in which evolves the dynamics of the system. This means that interested geometrical and dynamical characteristics of the underlying dynamics in the original phase space are preserved invariable in the reconstructed space as well.

Let $\mathbf{X}_{r}=\Phi^{(l)}(\mathbf{X})$ be the reconstructed phase space and $\boldsymbol{x}_{r}\left(t_{i}\right)=\Phi\left(\boldsymbol{x}\left(t_{i}\right)\right)$ the reconstructed trajectory for the embedding $\Phi$. Then the dynamics evolved in the original phase 
space is topologically equivalent to its mirror dynamical flow in the reconstructed phase space according to

$f_{r}^{t}\left(\boldsymbol{x}_{r}\right)=\Phi(\boldsymbol{x}) \circ f^{t}(\boldsymbol{x}) \circ \Phi^{-1}\left(\boldsymbol{x}_{r}\right)$

of the reconstructed phase space $\mathbf{X}_{r}$. In other words, the embedding $\Phi$ is a diffeomorphism which takes the orbits $f^{t}(\boldsymbol{x})$ of the original phase space to the orbits $f_{r}^{t}\left(\boldsymbol{x}_{r}\right)$ in the reconstructed phase in such a way of preserving their orientation and other topological characteristics as eigenvalues, Lyapunov exponents, or dimensions of the attractors. According to the above theory, in the reconstructed phase space we can estimate geometrical characteristics as dimensions, which correspond to the degrees of freedom of the underlying dynamics of the experimental time series, as well as dynamical characteristics as Lyapunov exponent, mutual information and predictors (Pavlos et al., 1999a,b). Moreover, is shown elsewhere that the method of reconstructed phase space conserve its significance even when the observed signal is derived by a stochastic process. (Argyris et al., 1998; Pavlos et al., 1999c).

\subsection{Correlation dimension}

The theoretical concepts described above permit us to use experimental time series in order to extract useful geometric characteristics, which provide information about the underlying dynamics. Such a characteristic is the correlation dimension $D$ defined as

$D=\lim _{r \rightarrow 0} \frac{d[\ln C(r)]}{d[\ln r]}$

where $C(r)$ is the so-called correlation integral for a radius $r$ in the reconstructed phase space. When an attracting set exists then $C(r)$ reveals a scaling profile

$C(r) \sim r^{d} \quad$ for $\quad r \rightarrow 0$.

The correlation integral depends on the embedding dimension $m$ of the reconstructed phase space and is given by the following relation

$C(r, m)=\frac{2}{N(N-1)} \sum_{i=1}^{N} \sum_{j=i+1}^{N} \Theta(r-\|\boldsymbol{x}(i)-\boldsymbol{x}(j)\|$,

where $\Theta(a)=1$ if $a>0$ and $\Theta(a)=0$ if $a \leq 1$, and $N$ is the length of the time series. The scaling exponent $d(m)$ increases as we increase the embedding dimension $m$. When the time series is related to a low-dimensional dynamical system, then $d(m)$ saturates at a final value $D$ for a sufficiently large embedding dimension $m_{0}$. Theoretically, the value $m_{0}$ is the smallest integer larger than $D$ according to Ding et al., (1993), but in practice, $m_{0}$ may attain larger values (Kugiumtzis, 1996). That is, an efficient embedding may require a larger $m$ than the smallest integer larger than $D$.

For periodic attractors the correlation dimension $D$ becomes equal to the topological dimension $d$ of the manifold M, which includes the attractor. Usually for a strange attractor, $D$ obtains a fractal value.
When the slopes $d(m)$ of the correlation integrals reveal plateaus at low values of $r$ and the plateau converges for increasing $m$, then this is strong evidence for low-dimensionality of the underlying dynamics to the observed signal. The stochastic component behaving as noise in the experimental time series, destroy the plateau and saturation profile at low values of the radius $r$ and makes the derivation of reliable dimension estimates difficult (Pavlos et al., 1999c).

\subsection{The method of surrogate data}

According to the relation (3) the scaling properties $C(r) \sim$ $r^{d}$ of the correlation integral as $r \rightarrow 0$ and the saturation of the scaling exponent, $d(m) \rightarrow D$, as $m$ increases are necessary conditions for the existence of low-dimensional dynamics underlying to the experimental time series. However, it has been shown that these conditions are not efficient in order to conclude low-dimensional dynamics from an experimental time series with broadband power spectrum, as they can be reached also by stochastic systems (Osborne and Provenzale, 1989; Provenzale et al., 1991). Moreover, according to Theiler (1991), the concept of low correlation dimension (fractal or integer) can be applied to time series in two distinct ways. The first one indicates the number of degrees of freedom in the underlying dynamics and the second quantifies the self-affinity or "crinkliness" of the trajectory through the phase space. In the first case, the scaling and saturation profile are caused by the recurrent character of the reconstructed trajectory, i.e. by uncorrelated in "time" and correlated in "space" state points. In the second case, they are caused by time correlated state points that are uncorrelated in space. In order to discriminate between the two cases, known as dynamic and geometric low-dimensionality, we restrict the sum in (5) to pairs $(\boldsymbol{x}(i), \boldsymbol{x}(j))$ with $|i-j|>w$, for the Theiler's parameter $w$ larger than the decorrelation time of the time series.

When low-dimensionality is persistent as a dynamic characteristic after the application of Theiler's criterion, then we have to decide first between linearity and nonlinearity and then between chaoticity and pure stochasticity. By the term chaoticity we mean the case that the deterministic component of the process is prevalent and reveals low-dimensional chaos. For a stochastic process, the deterministic component may correspond to low-dimensional, even nonlinear and chaotic dynamics, but its effect can hardly be observed as the process is driven mainly by noise. Therefore, we focus here on the solution to the first problem, i.e. determining whether the AE index time series is linear or nonlinear. This is done by following the method of "surrogate" data (Theiler et al., 1992a, 1992b).

The method of "surrogate" data includes the generation of an ensemble of data sets which are consistent to a null hypothesis. According to Theiler (1992a), the first type of null hypothesis is the linearly correlated noise which mimics the original time series in terms of autocorrelation function, variance and mean. The second and more general null hypothesis takes into account that the observed time series may be 
a nonlinear monotonic static distortion of a stochastic linear signal.

Every Gaussian process is linear while a non-Gaussian process can be linear or nonlinear. An experimental time series may show nonlinearity in terms of a non-Gaussian point distribution, which may be due to a nonlinear measurements function of a linear underlying dynamics. In this case, the generated "nonlinear" surrogate data mimic the original time series $x(i)$ in terms of autocorrelation function and propability density function $p(x)$. It is always possible for a nonperiodic time series of finite length to be a particular realization of a noise process or of a low-dimensional deterministic process. Therefore, it is a statistical problem to distinguish a nonlinear deterministic process from a linear stochastic process. For this purpose we use as a discriminating statistic a quantity $Q$ derived by a method sensitive to nonlinearity, as the correlation dimension estimation. The discriminating statistic $Q$ is calculated for the original and the surrogate data and the null hypothesis is verified or rejected according to the value of "sigmas" $S$

$S \equiv \frac{\left|\mu_{\mathrm{obs}}-\mu_{\mathrm{sur}}\right|}{\sigma_{\text {sur }}}$

where $\mu_{\text {sur }}$ and $\sigma_{\text {sur }}$ is the mean and standard deviation of $Q$ on the surrogate data and $\mu_{\mathrm{obs}}$ is the mean of $Q$ on the original data. For a single time series, $\mu_{\text {obs }}$ is the single $Q$ value (Theiler et al., 1992a).

The significance of the statistics is a dimensionless quantity, but we follow here the common parlance and we report it in terms of the units of $S$ "sigmas". When $S$ takes values higher than $2-3$ then the probability that the observed time series does not belong to the same family with its surrogate data is higher than $0.95-0.99$, correspondingly.

For testing the second more general null hypothesis described above we can follow the algorithm of Theiler (Theiler, 1992a), as well as the algorithm of Schreiber and Schmitz (Schreiber and Schmitz, 1996). Both algorithms create stochastic signals which have the same autocorrelation and amplitude distribution as the original time series.

According to the first algorithm, a white Gaussian noise is reordered to match the rank of the original time series (this is to make the original time series Gaussian). Then the phases of this signal are randomized (to destroy any possible nonlinear structure). Finally, the original signal is reordered to match the rank of the above constructed coloured noise (to regain the original amplitude distribution). The derived shuffled time series is the surrogate time series.

The algorithm of Theiler was improved by Schreiber and Schmitz by a simple iteration scheme in order to strengthen the ability of the surrogate data to fit more exactly the autocorrelation and power spectrum of the original time series. Starting with a white noise signal, its Fourier amplitudes are replaced by the corresponding amplitudes of the original data. The rank order of the derived stochastic signal is used to reorder the original time series. By this reordering, the matching of amplitude distribution is succeeded, but the matching of power spectrum achieved in the first step is altered. Therefore, the two step process is repeated several times until the change in the matching of power spectrum is sufficiently small. In this paper we follow the algorithm of Schreiber and Schmitz as it is more efficient to produce surrogate data which mimics the original data. For a comparison of the two algorithms see Pavlos et al. (1999a).

\subsection{Singular value analysis (SVD) and SVD reconstructed components of the original time series}

Singular value analysis has been proved to be a strong and effective method for modern time series analysis. It was used by Broomhead and King (1986) for first time and comes from the generalized theory of information. In this study we use the above analysis in two cases: (i) as a time series filter and (ii) to decompose a time series in its SVD reconstructed components which can be used for the detection of the underlying dynamics. Singular value analysis is applied to the trajectory matrix which is constructed by an experimental time series as follows:

$$
\begin{aligned}
\mathbf{X} & =\left[\begin{array}{c}
x\left(t_{1}\right), x\left(t_{1}+\tau\right), \ldots, x\left(t_{1}+(n-1) \tau\right) \\
x\left(t_{2}\right), x\left(t_{2}+\tau\right), \ldots, x\left(t_{2}+(n-1) \tau\right) \\
\ldots . \\
x\left(t_{N}\right), x\left(t_{N}+\tau\right), \ldots, x\left(t_{N}+(n-1) \tau\right)
\end{array}\right] \\
& =\left[\begin{array}{c}
\boldsymbol{x}_{1}^{T} \\
\boldsymbol{x}_{2}^{T} \\
\ldots \\
\boldsymbol{x}_{N}^{T}
\end{array}\right]
\end{aligned}
$$

where $x\left(t_{i}\right)$ is the observed time series and $\tau$ is the delay time for the phase space reconstruction. The rows of the trajectory matrix constitute the state vectors $\boldsymbol{x}_{i}^{T}$ on the reconstructed trajectory in the embedding space $\mathrm{R}^{n}$. As we have constructed $N$ state vectors in embedding space $\mathrm{R}^{n}$ the problem is how to use them in order to find a set of linearly independent vectors in $\mathrm{R}^{n}$ which can describe efficiently the attracting manifold within the phase space according to the theoretical concepts of paragraph 2.1. These vectors constitute part of a complete orthonormal basis $\left\{\boldsymbol{e}_{i}, i=1,2, . . n\right\}$ in $\mathrm{R}^{n}$ and can be constructed as a linear combination of vectors on the reconstructed trajectory in $\mathrm{R}^{n}$ by using the relation

$\boldsymbol{s}_{i}^{T} \mathbf{X}=\sigma_{i} \boldsymbol{c}_{i}^{T}$.

According to singular value decomposition (SVD) theorem it can be proved that the vectors $\boldsymbol{s}_{i}$ and $\boldsymbol{c}_{i}$ are eigenvectors of the structure matrix $\mathbf{X} \mathbf{X}^{T}$ and the covariance matrix $\mathbf{X}^{T} \mathbf{X}$ of the trajectory according to the relations

$\mathbf{X} \mathbf{X}^{T} \boldsymbol{s}_{i}=\sigma_{i}^{2} \boldsymbol{s}_{i}, \quad \mathbf{X}^{T} \mathbf{X} \boldsymbol{c}_{i}=\sigma_{i}^{2} \boldsymbol{c}_{i}$

(Brogan, 1982). The vectors $\boldsymbol{s}_{i}, \boldsymbol{c}_{i}$ are the singular vectors of $\mathbf{X}$ and $\sigma_{i}$ are its singular values, while the SVD analysis of $\mathbf{X}$ can be written as

$\mathbf{X}=\mathbf{S} \Sigma \mathbf{C}^{T}$ 
where $\mathbf{S}=\left[\boldsymbol{s}_{1}, \boldsymbol{s}_{2}, \ldots, \boldsymbol{s}_{n}\right], \mathbf{C}=\left[\boldsymbol{c}_{1}, \boldsymbol{c}_{2}, \ldots, \boldsymbol{c}_{n}\right]$ and $\mathbf{S}=$ $\operatorname{diag}\left[\sigma_{1}, \sigma_{2}, \ldots, \sigma_{n}\right]$. The ordering $\sigma_{1} \geq \sigma_{2} \geq \ldots \geq \sigma_{n} \geq 0$ is assumed. Moreover according to the SVD theorem the non-zero eigenvalues of the structure matrix are equal to nonzero eigenvalues of the covariance matrix. This means that if $n^{\prime}$ (where $n^{\prime} \leq n$ ) is the number of the non-zero eigenvalues, then $\operatorname{rank} \mathbf{X} \mathbf{X}^{T}=\operatorname{rank} \mathbf{X}^{T} \mathbf{X}=n^{\prime}$. It is obvious that the $n^{\prime}$-dimensional subspace of $\mathrm{R}^{N}$ spanned by $\left\{s_{i}, i=\right.$ $\left.1,2, \ldots, n^{\prime}\right\}$ is mirrored to the basis vector $\boldsymbol{c}_{i}$ which can be found as the linear combination of the delay vectors by using the eigenvectors $s_{i}$ according to (8). The complementary subspace spanned by the set $\left\{s_{i}, i=n^{\prime}+1, \ldots, N\right\}$ is mirrored to the origin of the embedding space $\mathrm{R}^{n}$ according to the same relation (8). That is, according to SVD analysis, the number of the independent eigenvectors $\boldsymbol{c}_{i}$ that are efficient for the description of the underlying dynamics is equal to the number $n^{\prime}$ of the non-zero eigenvalues $\sigma_{i}$ of the trajectory matrix. The same number $n^{\prime}$ corresponds to the dimensionality of the subspace containing the attracting manifold. The trajectory can be described in the new basis $\left\{\boldsymbol{c}_{i}, i=1,2, \ldots, n\right\}$ by the trajectory matrix projected on the basis $\left\{\boldsymbol{c}_{i}\right\}$ given by the product $\mathbf{X C}$ of the old trajectory matrix and the matrix $\mathbf{C}$ of the eigenvectors $\left\{\boldsymbol{c}_{i}\right\}$. The new trajectory matrix $\mathbf{X C}$ is described by the relation

$$
(\mathbf{X C})^{T}(\mathbf{X C})=\Sigma^{2}
$$

This relation corresponds to the diagonalization of the new covariance matrix so that in the basis $\left\{\boldsymbol{c}_{i}\right\}$ the components of the trajectory are uncorrelated. Also, from the same relation (11) we conclude that each eigenvalue $\sigma_{i}^{2}$ is the mean square projection of the trajectory on the corresponding $\boldsymbol{c}_{i}$, so that the spectrum $\left\{\sigma_{i}^{2}\right\}$ includes information about the extending of the trajectory in the directions $\boldsymbol{c}_{i}$ as it evolves in the reconstructed phase space. The explored phase space by the trajectory corresponds on the average to an $n$-dimensional ellipsoid for which $\left\{\boldsymbol{c}_{i}\right\}$ gives the directions and $\left\{\sigma_{i}\right\}$ the lengths of its principal evolves in the subspace spanned by eigenvectors $\left\{\boldsymbol{c}_{i}\right\}$ corresponding to non-zero eigenvalues. However, when the system is perturbed by external noise or deterministic external input, then the trajectory begins to be diffused also in directions corresponding to zero eigenvalues where the external perturbation dominates. As we show in the following, the replacement of the old trajectory matrix $\mathbf{X}$ with the new $\mathbf{X C}$ works as a linear low pass filter for the entire trajectory. Moreover, the SVD analysis permits one to reconstruct the original trajectory matrix by using the $\mathbf{X C}$ matrix as follows

$\mathbf{X}=\sum_{i=1}^{n}\left(\mathbf{X} \boldsymbol{c}_{i}\right) \boldsymbol{c}_{i}^{T}$

The part of the trajectory matrix which contains all the information about the deterministic trajectory as it can be extracted by observations corresponds to the reduced matrix

$$
\mathbf{X}_{d}=\sum_{i=1}^{n^{\prime}}\left(\mathbf{X} \boldsymbol{c}_{i}\right) \boldsymbol{c}_{i}^{T}
$$

which is obtained by summing only for the eigenvectors $\boldsymbol{c}_{i}$ with non-zero eigenvalues. From the relation (12) we can reconstruct the original time series $x(t)$ by using $n$ new time series $V\left(t_{i}\right)$ according to relation

$x(t)=\sum_{i=1}^{n} V_{i}(t)$

where every $V_{i}(t)$ is given by the first column of the matrix $\left(\mathbf{X} \boldsymbol{c}_{i}\right) \boldsymbol{c}_{i}^{T}$. The $V_{i}(t)$ time series are known as SVD reconstructed components (Elsner and Tsonis, 1996). This is a kind of $n$-dimensional spectral analysis of a time series.

The new time series $V_{i}(t)$ constitutes the reconstructed time series components of the SVD spectrum, corresponding to the spectrum of the singular vectors $\boldsymbol{c}_{i}$. The dependence of SVD analysis upon the existence of external noise is described by Broomhead and King (1986) for white noise and by Elsner and Tsonis (1996) for colored noise. In the case of white noise the singular values $\left\{\bar{\sigma}_{i}\right\}$ of $\mathbf{X}$ are shifted uniformly according to the relation

$\sigma_{i}^{2}=\bar{\sigma}_{i}^{2}+\left\langle\xi^{2}\right\rangle$

where $\bar{\sigma}_{i}$ are the eigenvalues of the unperturbed signal and $\left\langle\xi^{2}\right\rangle$ is the perturbation of the covariance matrix $\mathbf{X}^{T} \mathbf{X}$. Relation (15) indicates that in a simple case of white noise the existence of a non-zero constant background or noise floor in the spectrum $\left\{\sigma_{i}\right\}$ can be used to distinguish the deterministic component. In this way we can obtain the deterministic component of the observed time series

$\mathbf{X}_{d}=\sum_{\sigma_{i}>\text { noise }}\left(\mathbf{X} \boldsymbol{c}_{i}\right) \boldsymbol{c}_{i}^{T}$

where $\sigma_{i}$ corresponds to singular values above the noise background. Also the above relation (15) shows that in the case of white noise the perturbation of the singular values $\sigma_{i}$ is independent of them. In contrast, as we show in the following, in the case of colored noise the perturbation of the singular values is much stronger for the first singular value $\left\{\sigma_{1}\right\}$ than the others. This result could be expected as the colored noise includes finite dimensional determinism while the white noise is an infinite dimensional signal. The above difference between white and colored noise is significant because it makes the SVD analysis efficient to discriminate between different dynamical components of the original signal.

\section{SVD analysis of the AE index}

In this section we present the results of SVD analysis applied to the AE index in two different levels. First we use the SVD method as a filter of noise. In this case we estimate the correlation dimension of the AE index by transforming the original trajectory matrix. At this level we apply the method of surrogate data in order to decide between static and dynamic nonlinearity. In a second level we estimate the correlation dimensions and the singular values spectra of the SVD reconstructed components of the original time series. 
This permits one to distinguish between a high dimensional component and a low-dimensional component in the original time series of the AE index. The results of this study are used for a deeper understanding of the underlying $\mathrm{AE}$ time series dynamical process.

3.1 SVD filtering of the AE index time series and estimation of correlation dimension

The AE index describes the Auroral-zone magnetic activity which is related with the global magnetospheric dynamics through a complex system of currents. The magnetospheric dynamics during substorms is manifested as strong variability of the magnetospheric and ionospheric electric currents, especially the auroral electrojets (McPherron, 1995). Disturbances in the Earth's magnetic field produced by currents in the magnetosphere and ionosphere are commonly described by a number of magnetic activity indices, which are derived from certain physical parameters connected to the dominant phenomena causing the disturbance. The indices AU, AL, and $\mathrm{AE}$ give a measure of the strength of the auroral electrojets and are defined with the use of traces of the horizontal component $(\mathrm{H})$ of the geomagnetic field measured by a world-wide chain of auroral-zone magnetic observatories (Davis and Sugiura, 1966). AU is the maximum positive disturbance (upper envelope) recorded by any station in the chain. AL is the minimum disturbance defined by the lower envelope of the traces of the chain. AE is defined by the separation of the envelopes ( $\mathrm{AE}=\mathrm{AU}-\mathrm{AL})$ in order to obtain a better measure of the strength of the auroral electrojets.

Fig. 1a shows measurements of AE index which correspond to the second half of the year 1978. The sampling rate of the original signal was one minute while the time series used in this paper contains $N_{T}=32768$ data points that are the eight minute averages of the entire time series, rounded to the nearest power of two. That is, the original time series contains $N \cong 250.000$ data points. This time series has a much longer length than the time series used in our previous work (Pavlos et al., 1992b; 1994) as well as in the works of other scientists. The stationarity of the time series is tested by estimating the amplitude distribution for the first half of the data set and for the second half of it (see Fig. 1b). The amplitude distributions are the normalized ones as we subtract the mean and we have divided by the standard deviation. From the same figure it is obvious that the $\mathrm{AE}$ index time series reveals non-Gaussian amplitude distribution.

The random character of the AE time series is revealed by the decaying shape of the autocorrelation function (Fig. 1c) showing an abrupt decay during the first 100-200 minutes and a slow long decay afterwards. This profile of the autocorrelation function could be caused by two different mechanisms: a dynamical one, which corresponds to the abrupt decay, and a stochastic one (coloured noise), which is responsible for the slow decay. The two discontinuous lines in Fig. 1c reveal the two different mechanisms. Of course the abrupt decay cannot be explained solely as a chaotic behaviour as it is possible to be caused by a static nonlinear
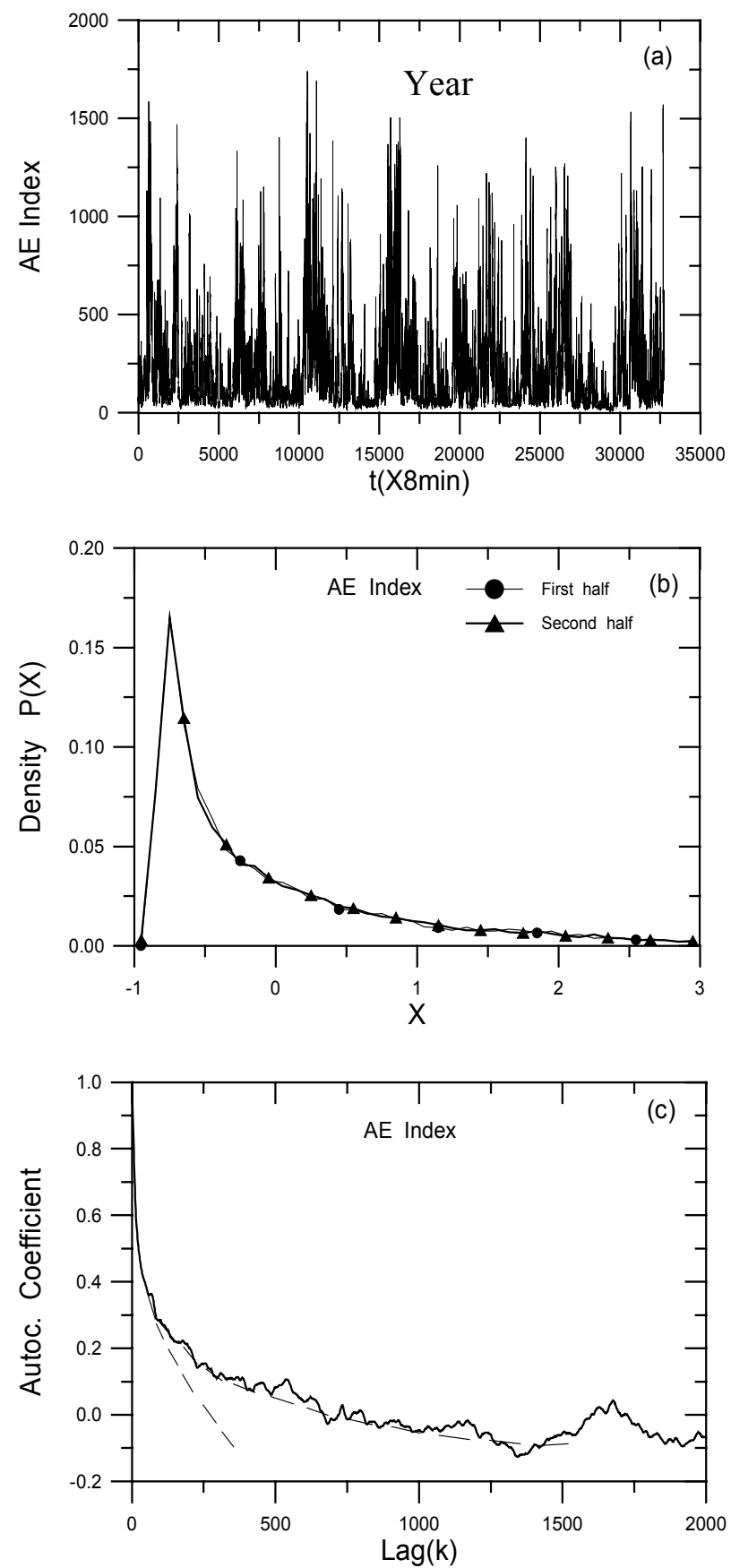

Fig. 1. (a) $\mathrm{AE}$ index measurements with eight minute time resolution corresponding to the second half of the year 1978. The bursting character of the $\mathrm{AE}$ index is obvious and indicates the strong coupling of the magnetosphere with the solar wind. (b) Amplitude distribution for the first and second half of the AE index time series. It is apparent the stationarity of $\mathrm{AE}$ index. (c) The autocorrelation coefficient for the first 2000 units of lag time indicates two different processes. The first corresponds to an abrupt decay of the autocorrelation coefficient and the second to a slow decay.

distortion of a linear stochastic system.

Fig. 2a shows the slopes $D$ of the correlation integrals estimated for embedding dimensions $m=4-20$. In this 

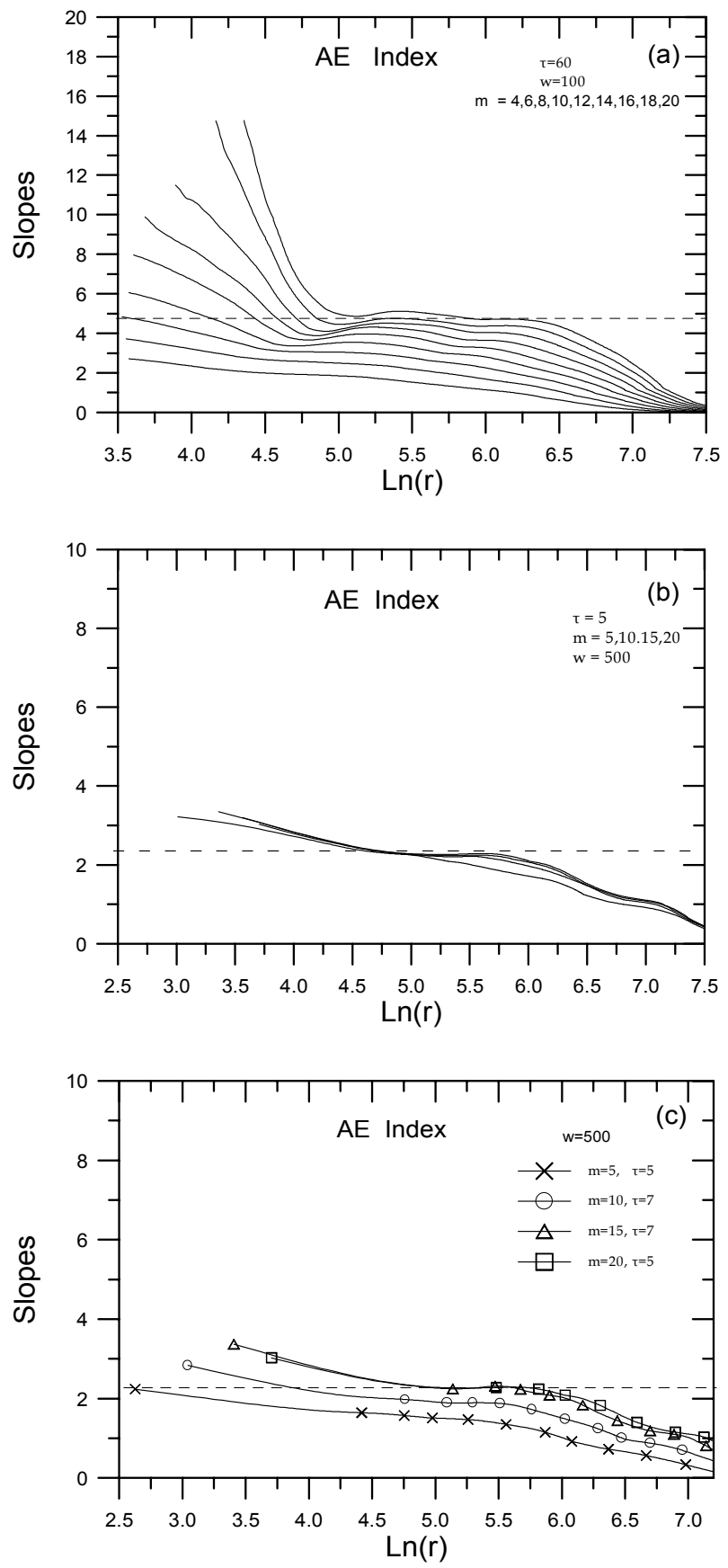

Fig. 2. (a) The slopes of the correlation integrals as a function of the radius $r$ estimated for embedding dimensions $m=4-20$, delay time $\tau=60$ units of sampling time and the Theiler's parameter $w=100$. (b) The same with (a) for $\tau=5, m=5-20$ and $w=500$ estimated by using the SVD filtering of the original signal and the same trajectory matrix for all embedding. (c) The same as (b) but now we have used independent trajectory matrices for every embedding.

estimation the Theiler's parameter takes the value $w=100$, while the slopes remain invariable for $w>100$, and for $\tau=$ $10-70$. An apparent plateau in a long region $\Delta \ln r \cong 5-$ 6.5 of the distance $r$ and a tendency for low value saturation of the slopes at the level $D \cong 5$ is also observed in Fig. 2a. The above low-dimensional profile is destroyed for lower distances $r(\ln r<5)$, something which indicates the strong component of the experimental perturbation of the system (see next sections).

In the following we estimate the slopes of the correlation integral by using the SVD method described in Section 2.4 for filtering the external noise. Fig. $2 b$ shows the slopes $D(r, m)$ of the correlation integrals $C(r, m)$ for embedding dimensions $m=5-20$ as a function estimated by using the trajectory matrix $\mathbf{X C}$ projected onto the basis $\left\{\boldsymbol{c}_{i}\right\}$. For this estimation we used columns included in the original trajectory matrix $\mathbf{X C}$ constructed for embedding dimension $m=20$. That is, for the embedding dimension $m=5$, the slope corresponds to the trajectory reconstructed by the first 5 columns of the $N \times 20$ matrix corresponding to the transformed matrix $\mathbf{X C}$ estimated for $m=$ 20. The same happens for the next values of $m$. Now it is obvious that there is a clear scaling of the correlation integral $C(r, m) \sim r^{d(m)}$ and a clear low value saturation of the slopes $d(r, m)=d[\ln C(r, m)] / d \ln r$, in a long region $\Delta \ln r \cong 4-6$ of the distance $r$. In this estimation the Theiler's parameter takes the value $w=500$ and the delay time was taken to be $\tau=5$. The dashed line indicates the low value saturation of the slopes and scaling exponents at the level $D \cong 2.5$. This result remains the same for dimensions $m>15$ in the case that the slopes are estimated for independent trajectory matrices. Fig. $2 \mathrm{c}$ is the same as Fig. $2 \mathrm{~b}$, but now we have reconstructed an independent matrix $\mathbf{X C}$ for each embedding which was used for the reconstruction of the corresponding trajectory. As we can see in Fig. $2 \mathrm{c}$ the saturation profile appears for $m>15$. The delay time $\tau$ for the construction of the trajectory matrix $\mathbf{X}$ was chosen appropriately for each embedding dimension. According to the embedding theory the number $d$ of degrees of freedom of the underlying dynamics corresponding to the $\mathrm{AE}$ index process is in the region $D+1 \leq d \leq 2 D+1$ with $D \cong 2.5$. The above saturation value $D \cong 2.5$ is sensibly lower than the value $D \cong 5$ estimated in Fig. 2 a and in our previous study (Pavlos et al., 1999a) where no SVD filter was used.

\subsection{Statistical test of the null hypothesis}

The non-Gaussian amplitude distribution of the AE index (shown in Fig. 1b) may imply the nonlinearity of the signal under appropriate conditions. However, the nonlinearity of a signal can be static or dynamic. As discussed in Section 2.3 a random like and non-Gaussian signal could be caused by a deterministic nonlinear and even chaotic underlying process or by a linear stochastic process observed through a nonlinear static distortion. Fig. 3a presents the slope of the correlation integral estimated in a 20-dimensional state space for the AE index and surrogate data. As in Section 3.1 we have used the XC trajectory matrix for the above estimation. Fig. $3 \mathrm{~b}$ presents the significance $(S)$ of the statistics as a function of $\ln r$. We observe that for small values of the distance (r) $\Delta \ln r \cong 4.5-5.5$ the significance stays within the region 

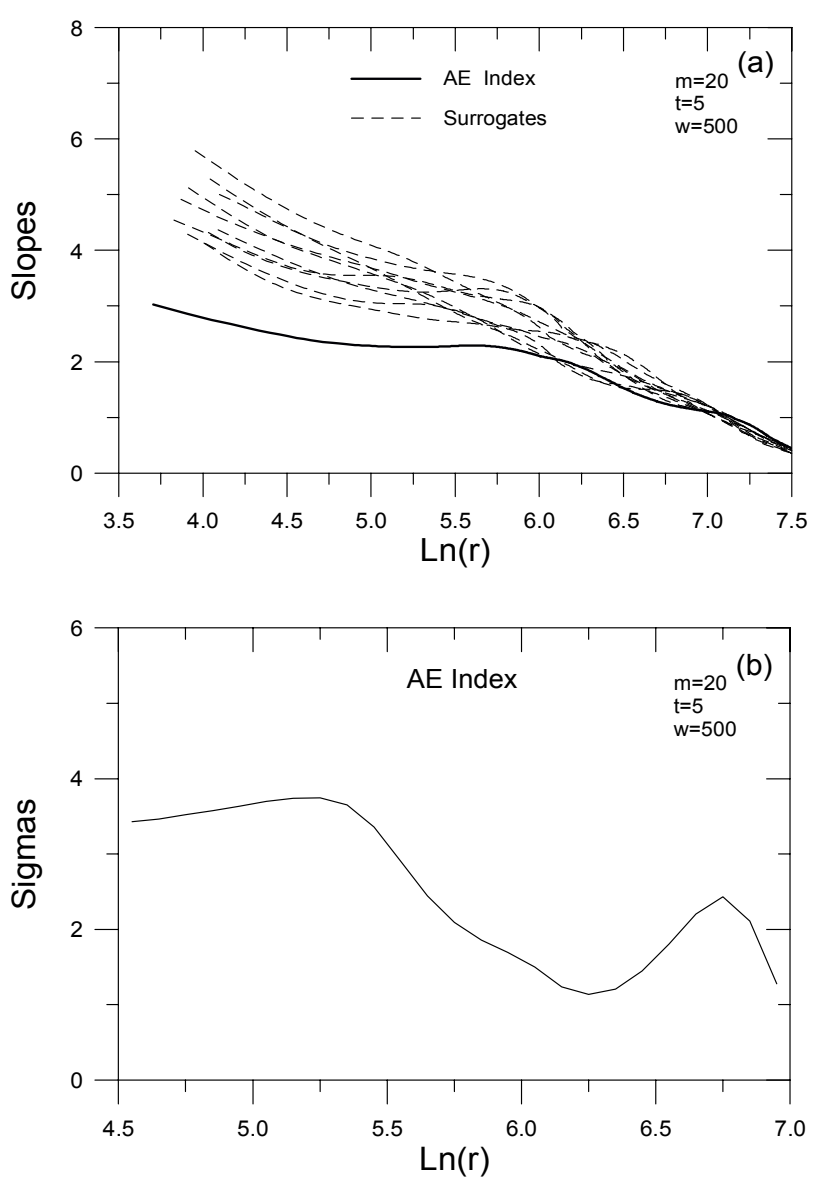

Fig. 3. (a) Slopes of the correlation integrals estimated for the original signal and its first 10 surrogate data as function of $\ln r$. (b) The significance of the statistics as function of $\ln r$, shown in (a).

$\sim 2-4$. This permits one to reject the null hypothesis with high confidence (>95\%). Comparing the above results with similar results obtained previously for the AE index (Pavlos et al., 1999a) we can conclude that the SVD filter is very helpful and strengthens the algorithm for uncovering lowdimensionality and dynamic nonlinearity of the underlying dynamical process to the original time series. In our previous paper (Pavlos et al., 1999a) we have estimated the same characteristics of the AE index but without applying the SVD filtering. There the significance of the statistics was found to take sensibly lower values than the ones found here.

\subsection{Correlation dimension and singular values for the SVD} reconstructed components of the $\mathrm{AE}$ index time series

In this subsection we study the slopes of the correlation integral and the singular values spectrum for the SVD reconstructed components $V_{i}(t)$ according to the theoretical concepts presented in Section 2.4. Figs. 4(b-d) show the reconstructed components $V_{1}(t), V_{4}(t), V_{10}(t)$ and Figs. 4(f-h) show the corresponding autocorrelation coefficients in comparison with the original time series and its autocorrelation coefficient (Figs. 4a, 4e). As we can see in this figure the
Table 1. This shows the ratio $\sigma_{2} / \sigma_{1}$ of the first two singular values of the $\mathrm{AE}$ index time series and its $V_{1}, V_{2-10} \mathrm{SVD}$ reconstructed components.

\begin{tabular}{|c|c|}
\hline & $\sigma_{2} / \sigma_{1}$ \\
\hline $\mathrm{AE}$ Index & 0.10 \\
\hline $\mathrm{AE}-V_{1}$ & 0.10 \\
\hline $\mathrm{AE}-\left(V_{2}-10\right)$ & 0.57 \\
\hline
\end{tabular}

amplitude of the values of the components are decreasing for the increasing values of the index $i$. The decorrelation times of the signals are also decreasing for increasing values of $i$. This reveals that the deterministic character of the components $V_{i}(t)$ weakens as the index $i$ increases. The above characters of the components $V_{i}(t)$ are in accordance with the fact that according to SVD analysis, every time series $V_{i}(t)$ corresponds to the projection of the trajectory on the eigenvectors $\boldsymbol{c}_{i}$ related to the $\sigma_{i}$ respectively (see relation 9). The eigenvalues $\sigma_{i}$ also decrease passing from low to high values of the index $i$, so that the amplitude of the extension of the trajectory along the axis $\boldsymbol{c}_{i}$ decreases as we go through from low to high values of $i$. By comparing Figs. $4 \mathrm{a}, 4 \mathrm{~b}$ and Figs. $4 \mathrm{e}, 4 \mathrm{f}$ we conclude that the first SVD component $V_{1}$ corresponds approximately to the trend of the original time series. At first glance the difference between $V_{1}$ and the other components is similar to the difference of a low and a high pass filter. However the relation between $V_{1}$ and the other components is more complicated as we show in the following. Fig. 5a shows the spectrum of the singular values $\sigma_{i}$ for the original time series. Fig. 5b is the same as Fig. 5a but estimated for the component $V_{1}(t)$ of the original time series and for embedding dimensions $m=10-25$. Figs. 5(a-b) reveal apparent similarity between the singular value spectra for both time series, the AE index and its first SVD component $V_{1}(t)$. Also we can notice the high difference between the value of the first eigenvalue $\sigma_{1}$ and the value of the next eigenvalues $\sigma_{i}, i=2-25$. Fig. 5c shows the spectrum of the singular values estimated for the time series

$V_{2-10}=\sum_{i=2}^{10} V_{i}(t)$

corresponding to the sum of ten first SVD reconstructed components except the component $V_{1}$. We can observe that the spectrum of the singular values is normal for the $V_{2-10} \mathrm{com}-$ ponent without any asymmetry in values of $\sigma_{i}$ observed for the $\mathrm{AE}$ index and its $V_{1}$ component. As Fig. 5c reveals clearly the existence of a noise background after the first $7-10$ singular values. Fig. $5 \mathrm{c}$ reveals that approximately the deterministic components of the SVD spectrum are the first $7-10$ components $V_{i}$, corresponding to nontrivial eigenvectors. Also Figs. 5(a-c) indicate a strong difference between the first component $V_{1}$ and the next SVD components of the $\mathrm{AE}$ index as we can conclude by studying the ratio $\sigma_{2} / \sigma_{1}$ of the first two singular values estimated for the $\mathrm{AE}$ index and its SVD components as shown in Table 1 . The ratio $\sigma_{2} / \sigma_{1}$ 

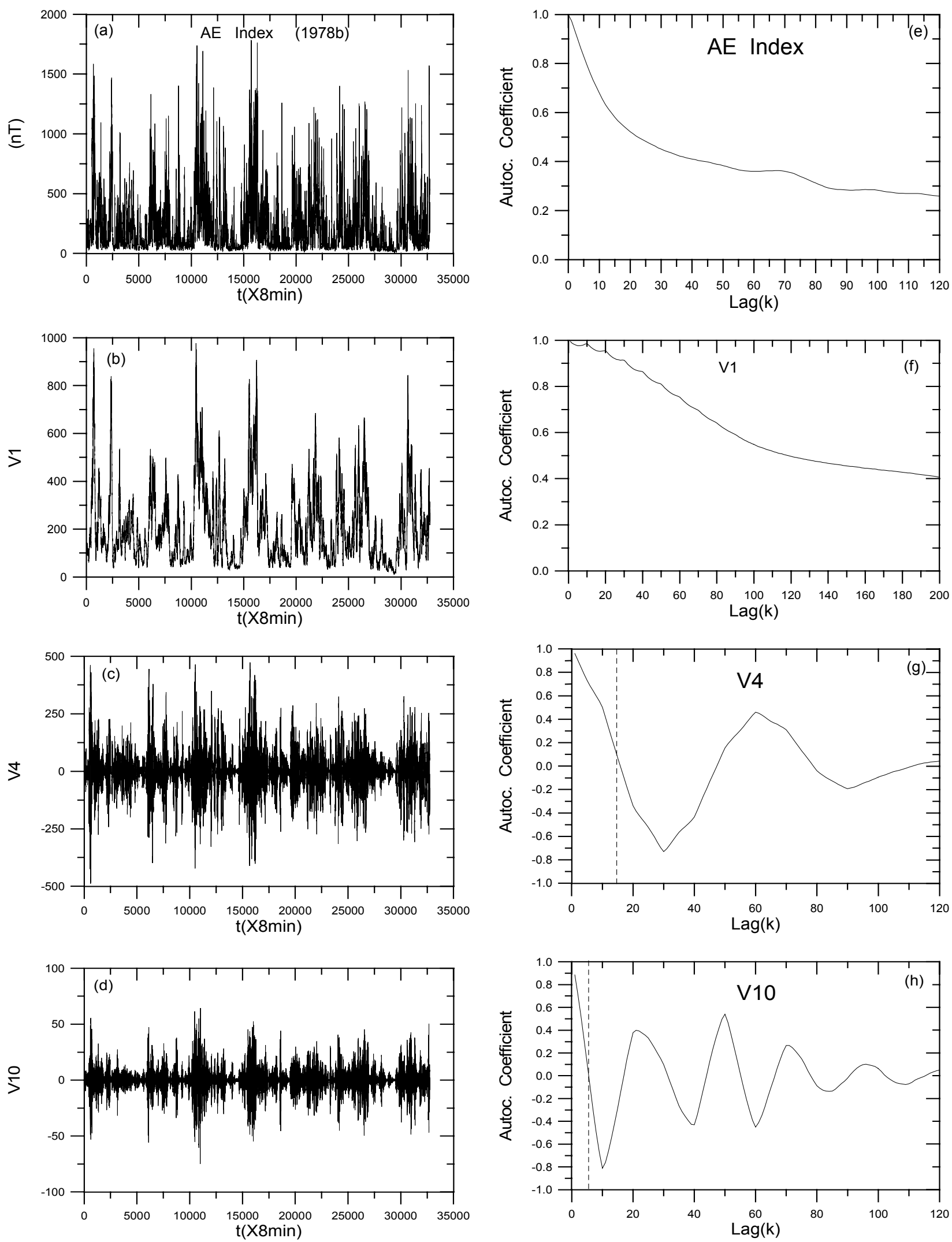

Fig. 4. (a) The AE index time series corresponding to the second semester of the year 1978. (b-d) The time series corresponding to the $V_{i}, i=1,4,10$, components of the SVD analysis of the signal shown in (a). (e-h) The autocorrelation coefficients estimated for the signals shown in (a-d) respectively. The dotted lines indicate when the autocorrelation function in $(\mathrm{g}, \mathrm{h})$ become zero for first time. 

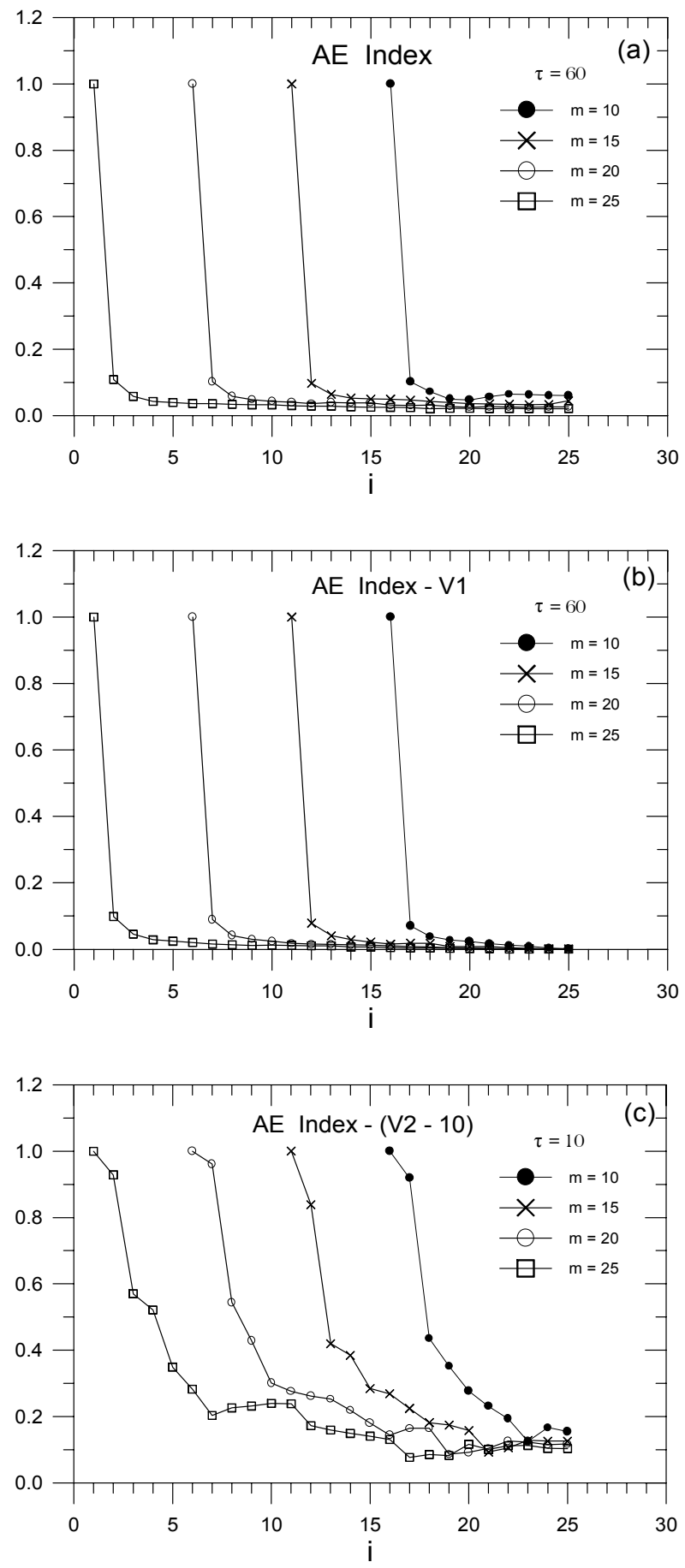

Fig. 5. (a-c) The spectra of singular values $\sigma_{i}, i=1-25$ estimated for the AE index and its SVD reconstructed components $V_{1}, V_{2-10}$.

takes small values $(\sim 0.1)$ both for the $\mathrm{AE}$ index and its $V_{1}(t)$ component but increases significantly for the case of the $V_{2-10}$ SVD components obtaining the value 0.57 . To understand the physical meaning of the above characteristics concerning the singular value spectrum of the AE index is a central point of this paper. In the following, we study the slopes of the correlation integrals estimated for $V_{1}$ and $V_{2-10}$
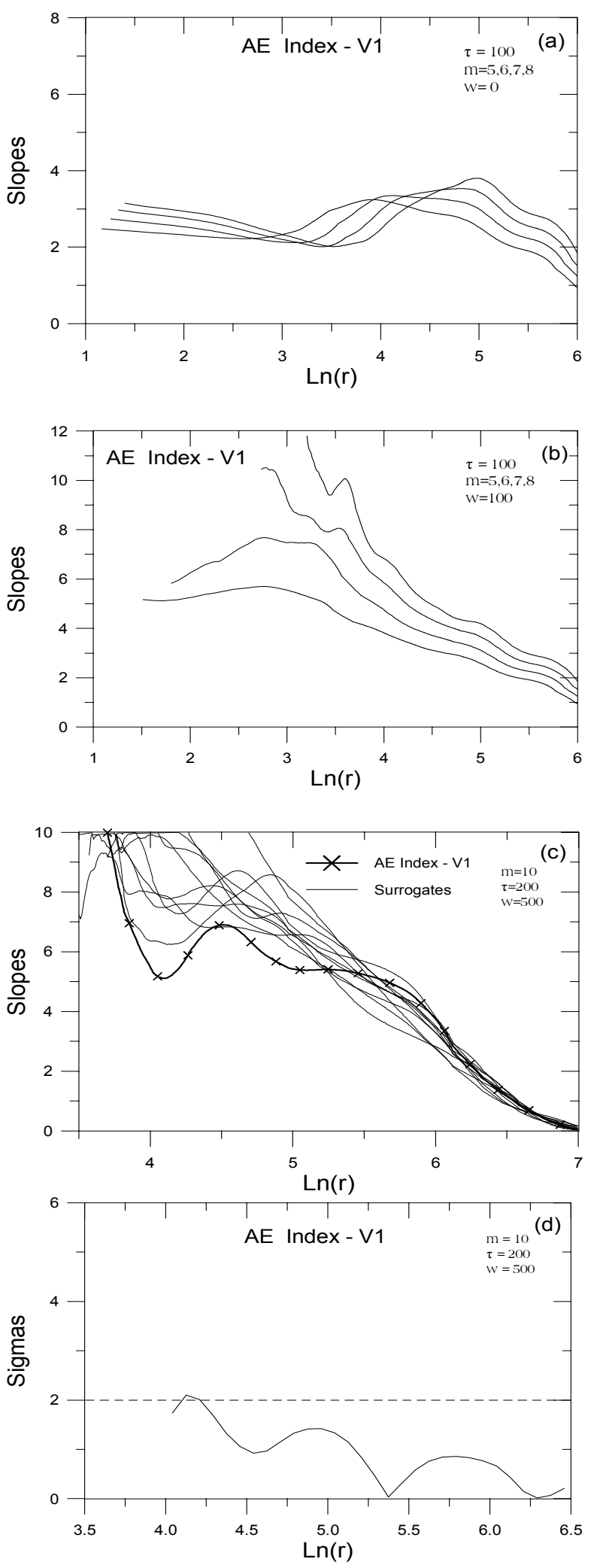

Fig. 6. (a) The slopes of the correlation integrals estimated for the $V_{1}$ SVD reconstructed component of the AE index for $m=5-$ $8, \tau=100$ and Theiler's parameter $w=0$. (b) The same as (a) estimated for $w=100$. (c) The same as (a) for AE index and its surrogate data and for parameters $m=10, \tau=200, w=500$. (d) The significance of the statistics shown in (c). 

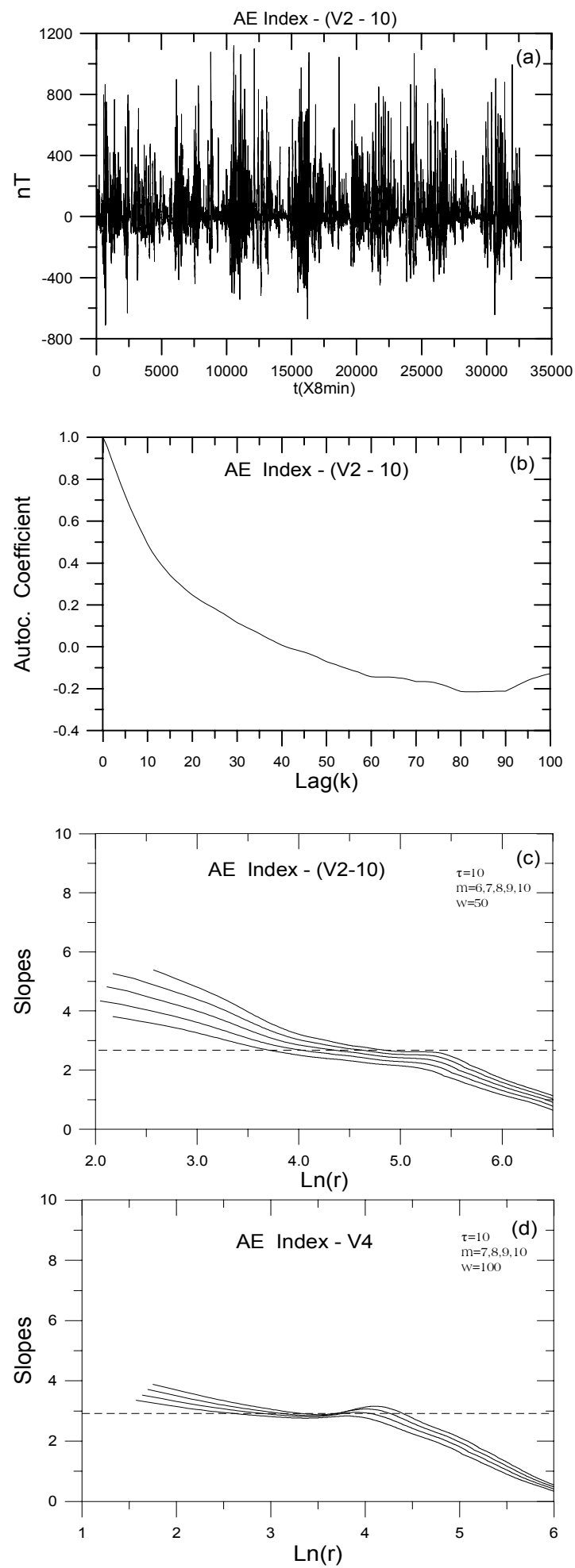

Fig. 7. (a) The time series corresponding to summarized SVD reconstructed component $V_{2-10}=\sum V_{i}(i=2-10)$, of the AE index signal. (b) The autocorrelation coefficient of the signal shown in (a). (c) The slopes of the correlation integral estimated for the $V_{2-10}$ SVD reconstructed component (shown in a) estimated for parameters $\tau=10, m=6-10, w=50$. (d) The same as (c) corresponding to the $V_{4}$ DVD reconstructed component of the AE index.
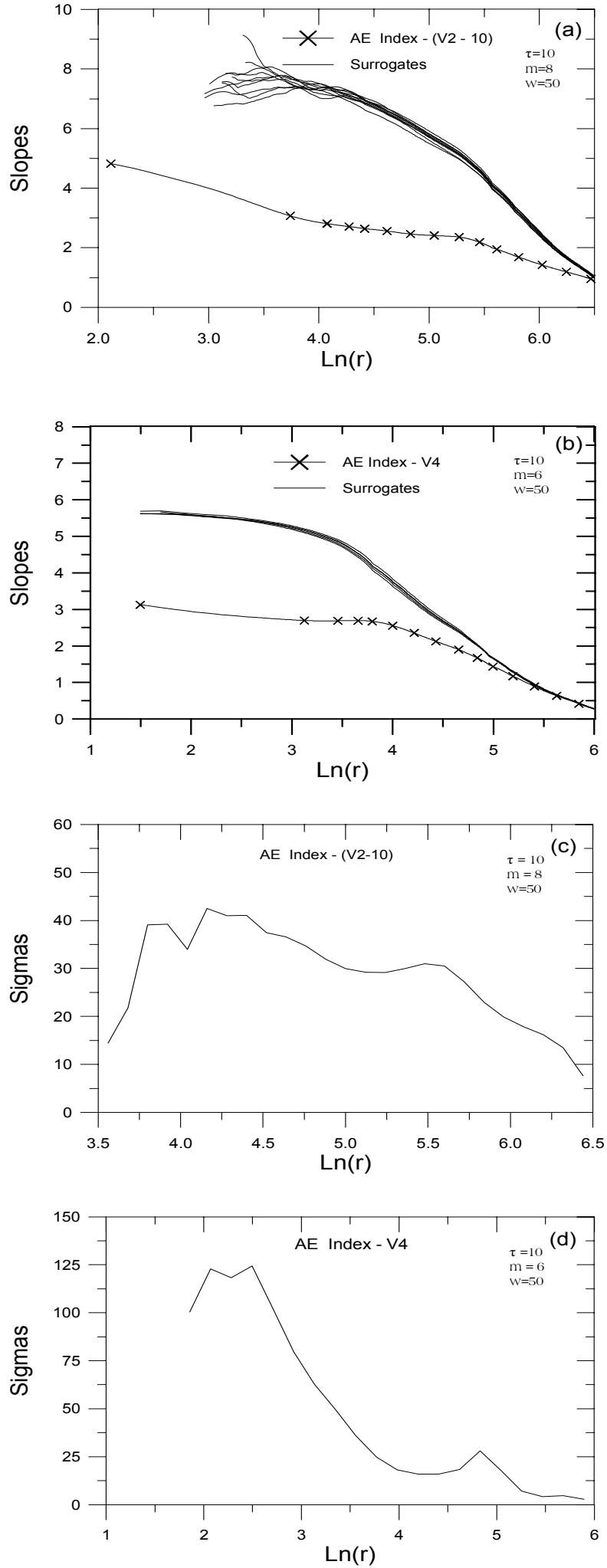

Fig. 8. (a,b) The slopes of the correlation integrals estimated for the $V_{2-10}, V_{4} \mathrm{SVD}$ reconstructed components of the AE index and their corresponding surrogate data. (c,d) The significance of the statistics shown in $(a, b)$ respectively. 
time series.

Figs. 6(a-b) show the slopes of the correlation integrals estimated for the first SVD component $V_{1}(t)$ of the AE time series. Fig. 6a corresponds to Theiler's parameter $w=0$ and Fig. $6 \mathrm{~b}$ to $w=100$. The first figure indicates a profile corresponding to low correlation dimension which, however, is destroyed when time correlated points of the reconstructed trajectory are excluded. This characteristic reveals that there is no low-dimensional deterministic process underlying to the first SVD component of the AE index. This point of view is strengthened by Figs. 6(c-d) which present the comparison with surrogate data. Fig. 6c shows the slopes of nonlinear surrogate data constructed for the $V_{1}$ time series in comparison with the slope of the $V_{1}$ component. As we can see in Fig. 6d the significance of the statistics indicates a small possibility for the discrimination between dynamic nonlinearity and static nonlinearity, taking values lower than two sigmas at the main part of the region of values of distance $r$.

Figs. 7(a-b) show the $V_{2-10}$ SVD component and its autocorrelation coefficient. The decorrelation time of this time series is $30-40$ lags. Fig. 7c shows the slopes of the correlation integrals for the $V_{2-10}$ time series, estimated for $m=6-10$ and $w=50$. This figure reveals a scaling character in the region $\Delta \ln r=4-5$ and a saturation of the scaling exponents at the value $D \cong 2.5$. Fig. $7 \mathrm{~d}$ shows the slopes of the correlation integrals estimated for the $V_{4}$ component of the SVD spectrum of the AE index, for $m=7-10$ and $w=100$. This figure also reveals a scaling profile and low value saturation of the scaling exponents.

Figs. 8(a-b) show the slopes of the nonlinear surrogates estimated for the $V_{2-10}$ and the $V_{4}$ components, in comparison with the slope of the original time series, for embedding dimension $m=8$ and $m=6$ respectively. The significance of the statistics coresponding to the Figs $8(a-b)$ is shown on Figs. 8(c-d). Figures $8(\mathrm{c}-\mathrm{d})$ clearly indicate the possibility for the rejection of the null hypothesis in both cases as the significance takes high values.

The previous results of the SVD analysis of the AE index reveal that the original time series includes a strong linear and high dimensional stochastic component which is related with the first component $V_{1}$ of the SVD spectrum of the reconstructed components. Moreover, the next SVD components $V_{i}, i>1$ indicate a low-dimensional and nonlinear underlying dynamical process.

\section{SVD analysis of the Lorenz system}

In this section we repeat the SVD analysis of the Section 3 applied to the Lorenz system in three stages: a) the Lorenz system without external noise b) the Lorenz system perturbed by white noise and c) the Lorenz system perturbed by colored noise. The result of the SVD analysis applied to a known dynamical system will help to understand at a deeper level the previous results of the SVD analysis for the AE index.

\subsection{Purely deterministic time series}

Fig. 9a shows the $x$-component time series of the Lorenz system obtained by arithmetic solution according to Abarbanel (1993). Figs. 9(b-c) show the $V_{1}$ and $V_{4}$ component of the spectrum of the SVD reconstructed components of the original time series shown in Fig. 9a and Fig. 9d shows the $V_{2-10}$ component corresponding to the sum $\sum V_{i}$ for $i=2-10$. Figs. 9(e-h) show the autocorrelation coefficients corresponding to the time series shown in Figs. 9(ad). We can notice here that the $V_{1}$ component includes high determinism with long decorrelation time. As the index $i$ increases the determinism decreases as we can conclude from the autocorrelation coefficient shown in Fig. 9g corresponding to the $V_{4}$ component. The component $V_{1}$ corresponds to the trend of the original time series (see Fig. 9b) while the next components correspond to the high frequencies. The component $V_{2-10}$ approximates efficiently the original time series as we can conclude from Fig. 9a and Fig. 9d.

Figs. 10(a-d) show the slopes of the correlation integrals corresponding to the time series shown in Figs. 9(a-d). The slopes of the surrogate data corresponding to the original time series and its SVD components are shown in Figs. 10(eh). From Figs. 10(a-d) we conclude that for the Lorenz system every SVD reconstructed component reveals a scaling and saturation profile similar to that shown by the original time series, as the correlation dimension of all the SVD components is almost equal to the correlation dimension of the original time series of the Lorenz system. That is the estimated correlation dimension for the $x(t)$ time series of the Lorenz system and the correlation dimension of its SVD component is $\sim 2.1-2.5$ as it can be concluded by Figs. 10(a-d). On the other hand, Figs. 10(e-h) indicate clearly the possibility for the discrimination of the linear stochastic data transformed by nonlinear static distortion and the original time series. This happens both for the Lorenz system as well as for its SVD components. Here we can notice strong dissimilarity between the Lorenz system and the system corresponding to the $\mathrm{AE}$ index especially concerning the first SVD component $V_{1}(t)$. As we have shown in Figs. 6(b-c) in the case of the $\mathrm{AE}$ index the $V_{1}$ component reveals a strong difference from the original time series concerning the slopes of the correlation integral. That is, for the $V_{1}$ component of the $\mathrm{AE}$ index there is no significant low-dimensional profile and no significant discrimination from the surrogate data.

Similar results we have also found in the case of the singular value spectrum. Figs. 11(a-c) show the normalized singular values $\left\{\sigma_{i}\right\}$ for the $x(t)$ time series of the Lorenz system and its $V_{1}, V_{2-10} \mathrm{SVD}$ components. The result here is similar to that of the slopes, as there is no significant difference between the $\left\{\sigma_{i}\right\}$ spectra estimated for the original time series and its SVD components. Contrary to the Lorenz system for the AE index system we observe a strong difference between the singular values spectra $\left\{\sigma_{i}\right\}$ corresponding to the $\mathrm{AE}$ index and its $V_{1}$ component compared to the SVD components of the AE index time series (see Figs. 5(a-c)). However, we have observed a strong similarity between the 

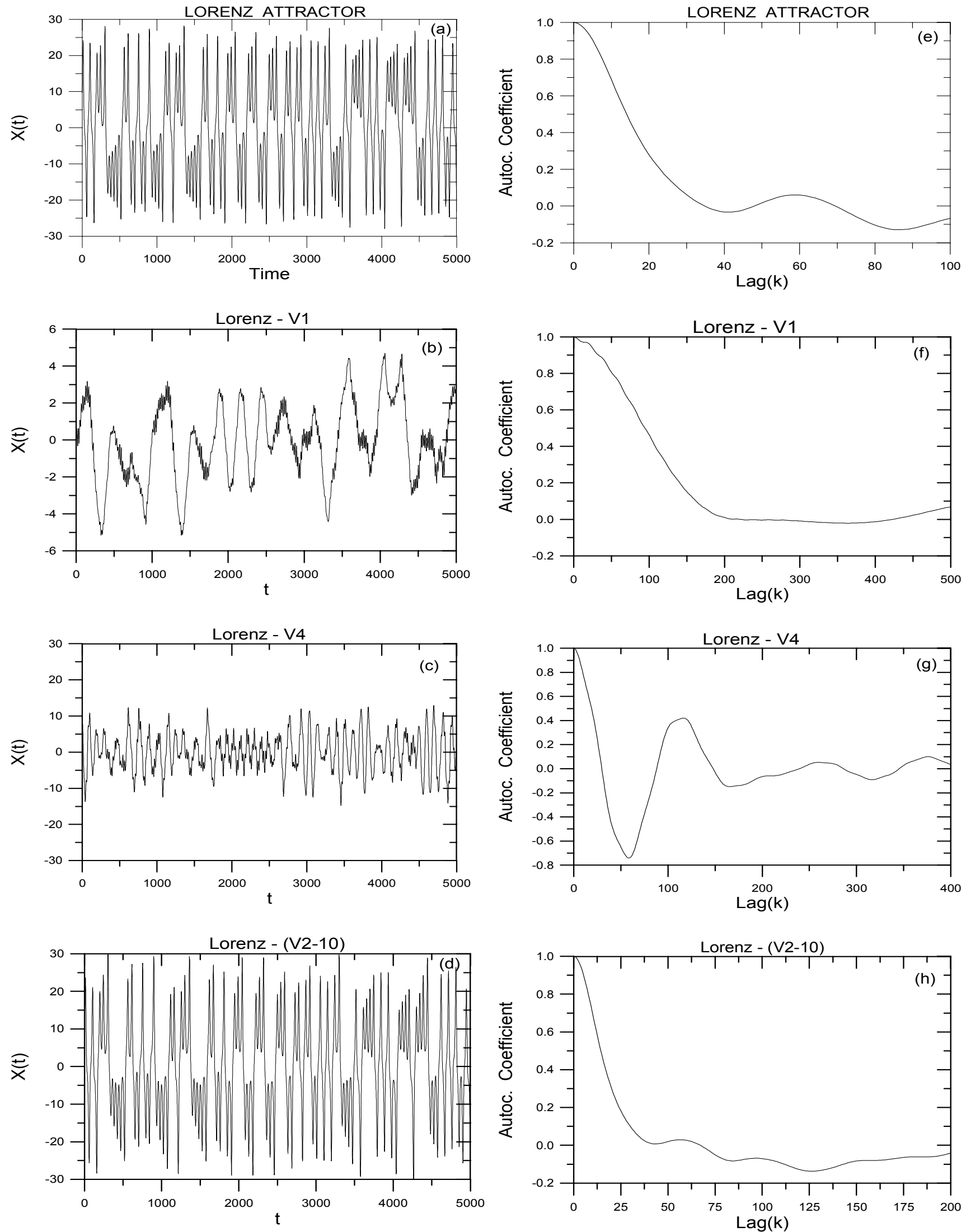

Fig. 9. The same with Fig. 4 corresponding to the Lorenz time series $x(t)$ and its SVD reconstructed components. 

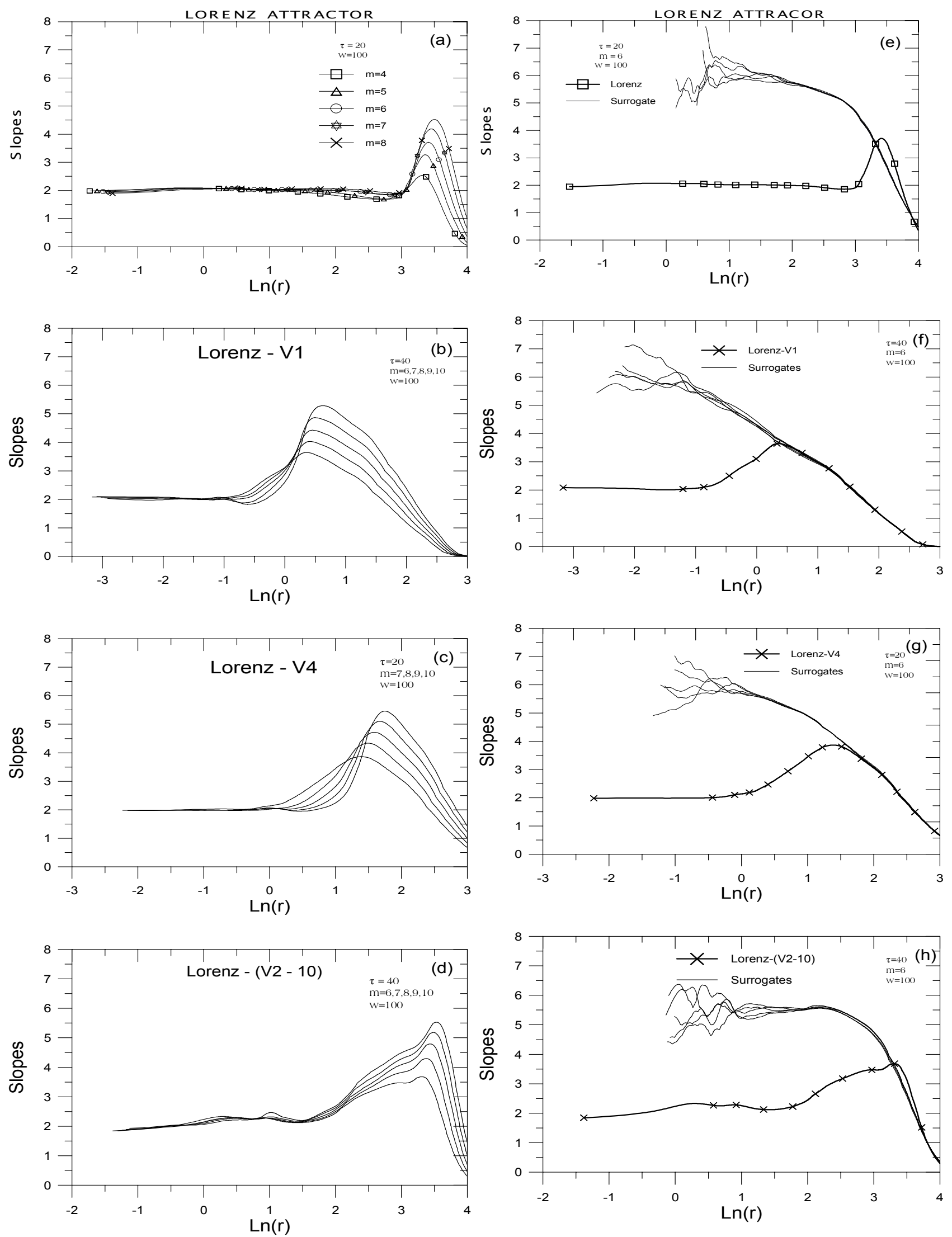

Fig. 10. (a-d) the slopes of the correlation integrals estimated for the Lorenz time series $x(t)$ and its SVD reconstructed components $V_{1}, V_{4}$, and $V_{2-10} \cdot(\mathbf{e}-\mathbf{h})$ The slopes of the correlation integrals estimated for the surrogate data corresponding to the $x(t)$ Lorenz time series and its SVD components and for embedding $m=6$. 

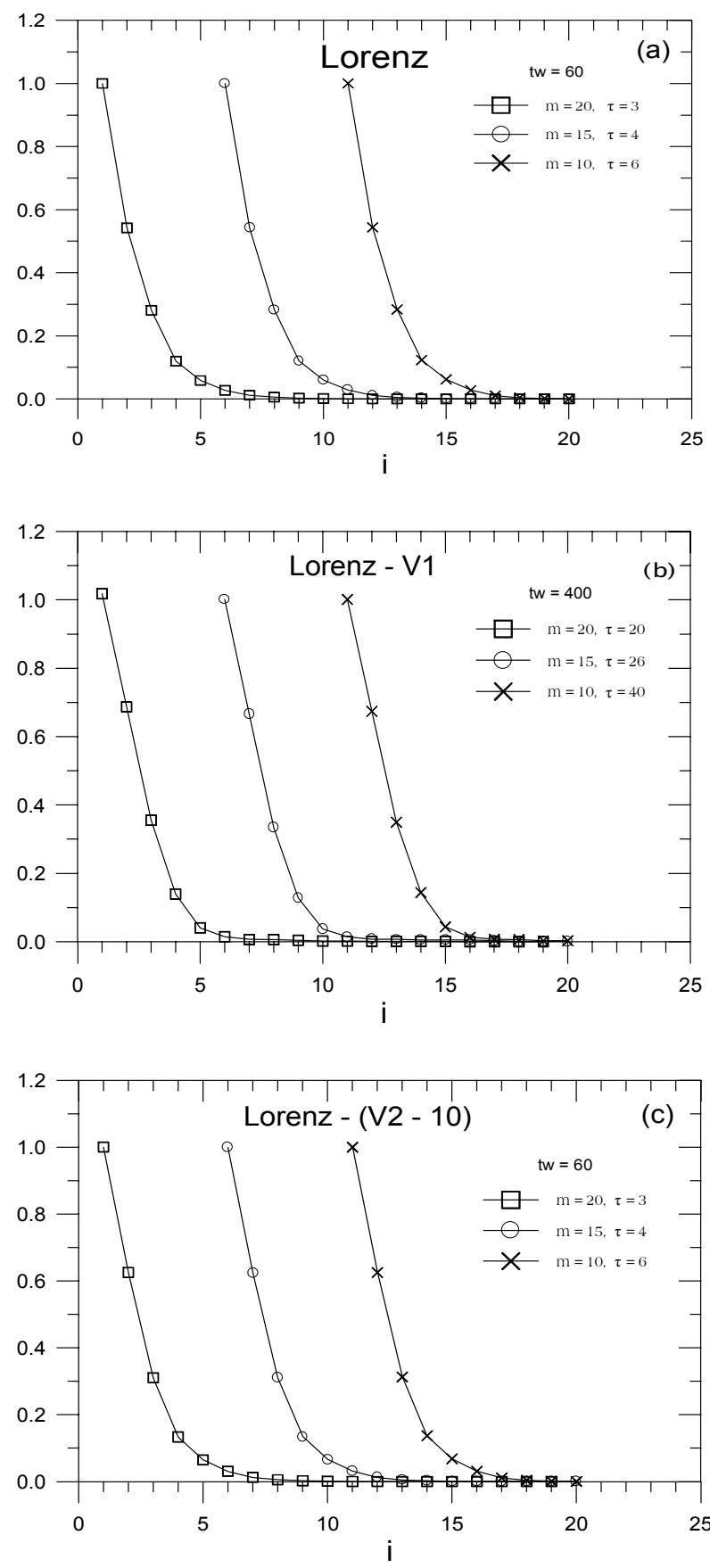

Fig. 11. Singular value spectra estimated for the $x(t)$ Lorenz time series and its SVD components.

spectrum $\left\{\sigma_{i}\right\}$ corresponding to the original AE index time series and the spectrum $\left\{\sigma_{i}\right\}$ estimated for the $V_{1}$ SVD component of the AE index. The above comparison of the spectra estimated for the AE index system and the Lorenz system becomes more evident by comparing Table 2 and Table 1 . Table 2 shows the ratio $\sigma_{2} / \sigma_{1}$ estimated for the Lorenz signal and its SVD components and indicates that the ratio $\sigma_{2} / \sigma_{1}$ is almost invariable passing from the original signal to its SVD components. However, for the $\mathrm{AE}$ index, the ratio $\sigma_{2} / \sigma_{1}$ was
Table 2. The same as Table 1 corresponding to the deterministic $x(t)$ Lorenz signal.

\begin{tabular}{|c|c|}
\hline & $\sigma_{2} / \sigma_{1}$ \\
\hline Lorenz & 0.54 \\
\hline Lorenz $-V_{1}$ & 0.68 \\
\hline Lorenz- $\left(V_{2-10}\right)$ & 0.63 \\
\hline
\end{tabular}

found to be invariable passing from the original signal to its $V_{1}$ component and increases sensibly passing from $V_{1}$ to the $V_{2-10}$ SVD component.

Summarizing the results of the SVD analysis applied to the Lorenz system, we can conclude the following: For purely dynamical systems the correlation dimension estimated by the slopes of the correlation integrals and the singular value spectrum remains invariable as we pass from the original time series to its SVD reconstructed components. However, in the case of an experimental time series corresponding to an unknown underlying process, it is possible to show strong differences as we pass from the original time series to its first SVD component and then to the its next components.

In order to go deeper for a physical explanation of these characteristics we repeat the above SVD analysis for the Lorenz system perturbed by external noise.

\subsection{Perturbation by external white noise}

In this section we study the $x$-time series of the stochastic Lorenz system perturbed by external additive white noise according to the relation

$x(t)=x_{L}(t)+e x_{\mathrm{wn}}(t)$

where the $x_{\mathrm{wn}}(t)$ time series was obtained to have mean value zero and standard deviation one.

We use time series corresponding to the parameters $e=$ $0.1,0.3,0.5,1.0,5.0$. The respective percentages of the white noise to the above values are: $0.78 \%, 2.34 \%, 3.9 \%$, $7.8 \%, 39 \%$.

Fig. 12a shows the $x$-Lorenz time series obtained for $39 \%$ external white noise. Comparing this figure with Fig. 9a we notice that, although the perturbation is strong enough, the stochastic time series conserves the general profile of the original time series. Fig. 12b shows the autocorrelation coefficient for the purely deterministic Lorenz time series and for the corresponding stochastic Lorenz time series. As we notice in this figure the white noise does not perturbs strongly the autocorrelation coefficient. Especially at high values of lag the white noise leaves invariant the autocorrelation coefficient even for $39 \%(e=5)$ percentage of noise. Fig. 12c presents the slopes of the correlation integrals estimated for the stochastic Lorenz time series $(e=0.1-5.0)$ and the original Lorenz time series $(e=0)$ for embedding dimension $m=6$, delay $\tau=10$ and Theiler's parameter $w=100$. In this figure we notice a significant characteristic that has 

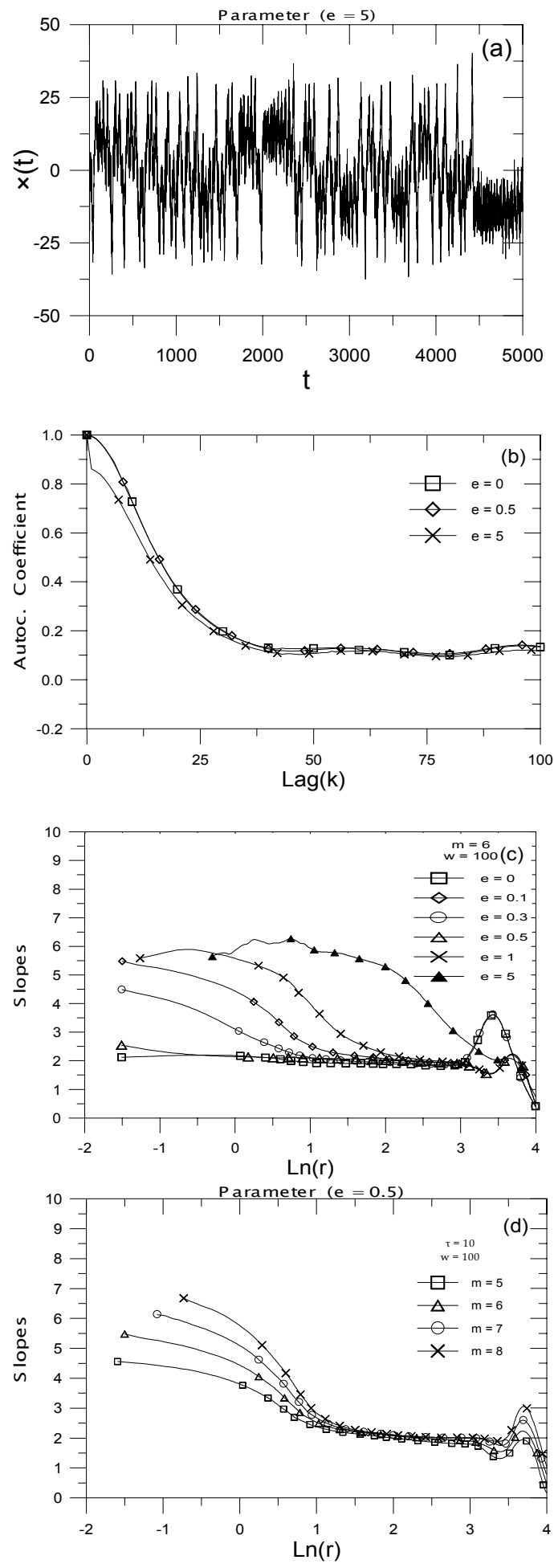

Fig. 12. (a) The $x(t)$ Lorenz signal perturbed by external additive white noise corresponding to the value parameter $e=5$ (see the text). (b) Autocorrelation coefficient of the purely deterministic Lorenz signal as well as of the two stochastic Lorenz signals $(e=0.5,5)(\mathbf{c}, \mathbf{d})$ Slopes of the correlation integrals of the $x(t)$ deterministic Lorenz signal and of $x(t)$ stochastic Lorenz signals corresponding to different levels of white noise perturbation. The percentage of white noise for every parameter $e$ value is $0.78 \%(e=0.1), 2.34 \%(e=0.3), 3.9 \%(e=0.5), 7.8 \%(e=$ $1), 39 \%(e=5)$. been analyzed extensively in our previous paper (Pavlos et al., 1999c). As we can see in Fig. 12c the slope of the correlation integral is destroyed gradually from low to high values of $\ln r$ as we increase the percentage of the stochastic external perturbation. Fig. 12d shows the slopes for embedding dimensions $m=5-8$ estimated also for the case of $3.9 \%$ $(e=0.5)$ of external white noise perturbation. For distance $r: \ln r<1$ there is no scaling and no saturation profile of the slopes. However, for higher values of the distance $r$ in the reconstructed phase space, the slopes remain invariable revealing the same scaling and saturation profile with the unperturbed and purely deterministic original time series $x(t)$ of the Lorenz system.

Fig. 13a shows the stochastic $x(t)$ Lorenz time series perturbed by $7.8 \%(e=1)$ external additive white noise, while Figs. 13(b-d) correspond to the $V_{1}, V_{4}$ and $V_{2-15}$ SVD components of the original signal shown in Fig. 13a. The $V_{2-15}$ component corresponds to the sum $\sum V_{i}, i=2-15$. The slopes of the correlation integrals corresponding to the time series shown in Figs. 13(a-d), are presented in Figs. 13(e-h). The slopes of the original stochastic Lorenz $(e=1)$ time series, shown in Fig. 13e reveal scaling and saturation profile only at high values of the $\ln r$, in the region $\Delta \ln r=2-3$. The saturation value is $D \cong 2.0-2.1$ and is approximately equal to the correlation dimension of the purely deterministic $x$-Lorenz time series. This characteristic remains the same for the first $\left(V_{1}\right)$ SVD component of the stochastic $(e=1)$ Lorenz time series as we can see in Fig. 13f. However, the scaling and saturation profiles are now observed at the region $\Delta \ln r=0-1$ of the distance $r$. The same result was obtained for the $V_{4}$ and $V_{2-15}$ components but now the saturation value $D$ of the scaling exponents was found to take little higher values, as shown in Fig. 13g and Fig. 13h.

Figs. 14(a-b) show the autocorrelation coefficient of the SVD components $V_{1}, V_{4}$ and $V_{2-15}$ corresponding to the purely deterministic $x$-Lorenz time series and the stochastic $x$-Lorenz time series corresponding to the strong perturbation by percentage 39\% (e=5) of external additive white noise. It is apparent here that the strong component of the external white noise leaves invariant the autocorrelation coefficients in all the cases of the original time series (see Fig. $12 \mathrm{~b})$ as well as of its SVD components (Figs. 14(a-b)). Fig. $14 \mathrm{c}$ shows a comparison of the autocorrelation coefficients corresponding to the stochastic $(e=1) x$-Lorenz time series and its SVD components. This figure reveals that the decorrelation time of the $V_{1}$ component is longer than that of the original signal, while the decorrelation time of the $V_{4}$ component is shorter than that of the $V_{2-15}$ component. Also, the decorrelation time of the original signal is longer than its $V_{2-15}$ and $V_{4}$ SVD components. These characteristics are similar to those of the AE index and the purely deterministic $x$-Lorenz signal (see Figs. 4(e-h), 9(e-h)).

Figs. 15(a-b) show the spectrum of the singular values $\left\{\sigma_{i}\right\}, i=1-20$ estimated for the purely deterministic $(e=$ 0) $x$-Lorenz system and the two stochastic Lorenz time series $(e=1,5)$ caused by external additive white noise perturbation as well as for their SVD components $V_{1}$ and $V_{2-15}$. 

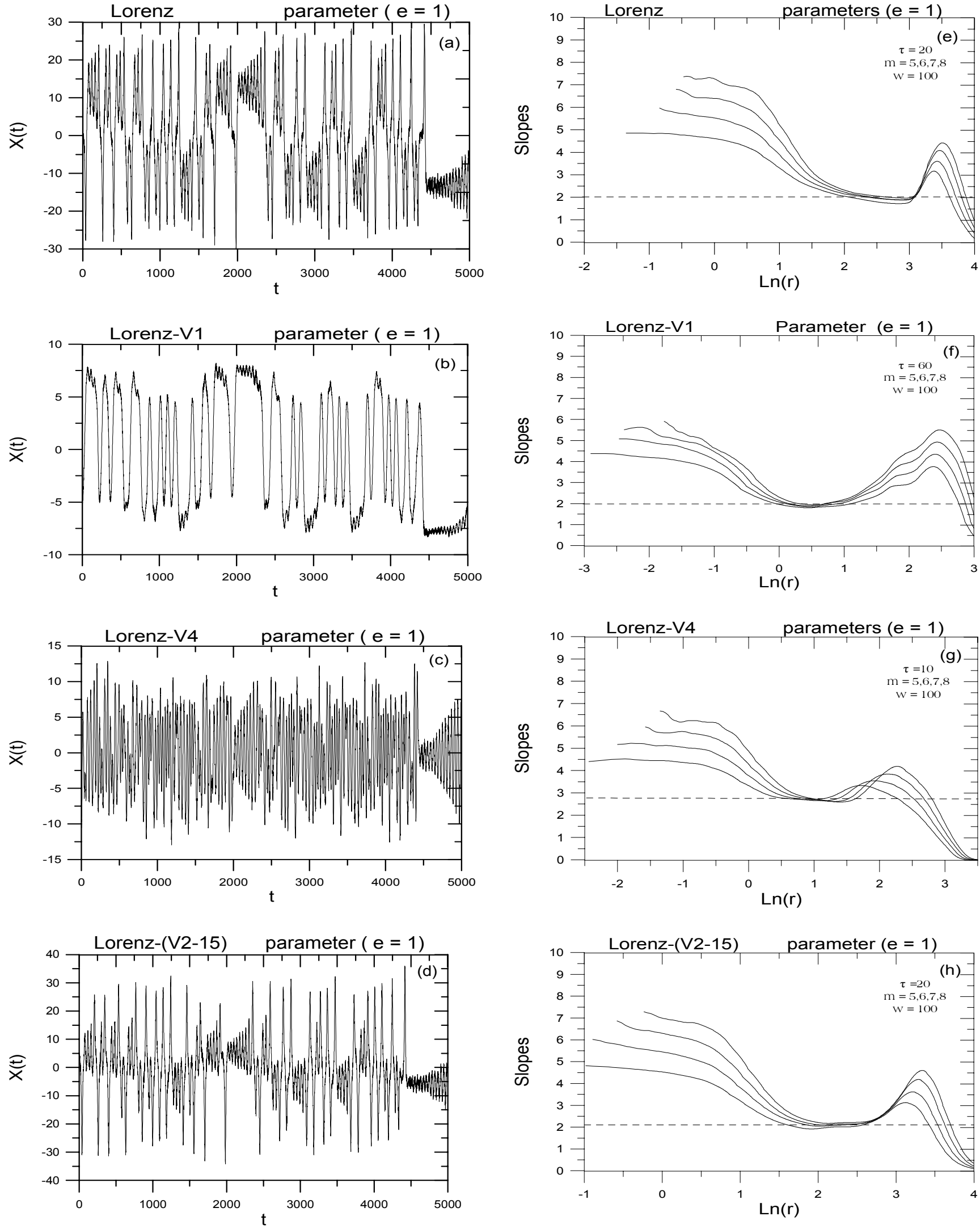

Fig. 13. (a-d) The stochastic Lorenz signal corresponding to the white noise perturbation $7.8 \%(e=1)$ and its SVD reconstructed components $V_{1}, V_{4}, V_{2-15}$. The last signal $V_{2-15}$ corresponds to the summarized SVD component $\sum V_{i}, i=2-15$. (e-h) The slopes of the correlation integrals estimated for the signals shown in (a-d) correspondingly embedding $m=5-8$ and $w=100$. 

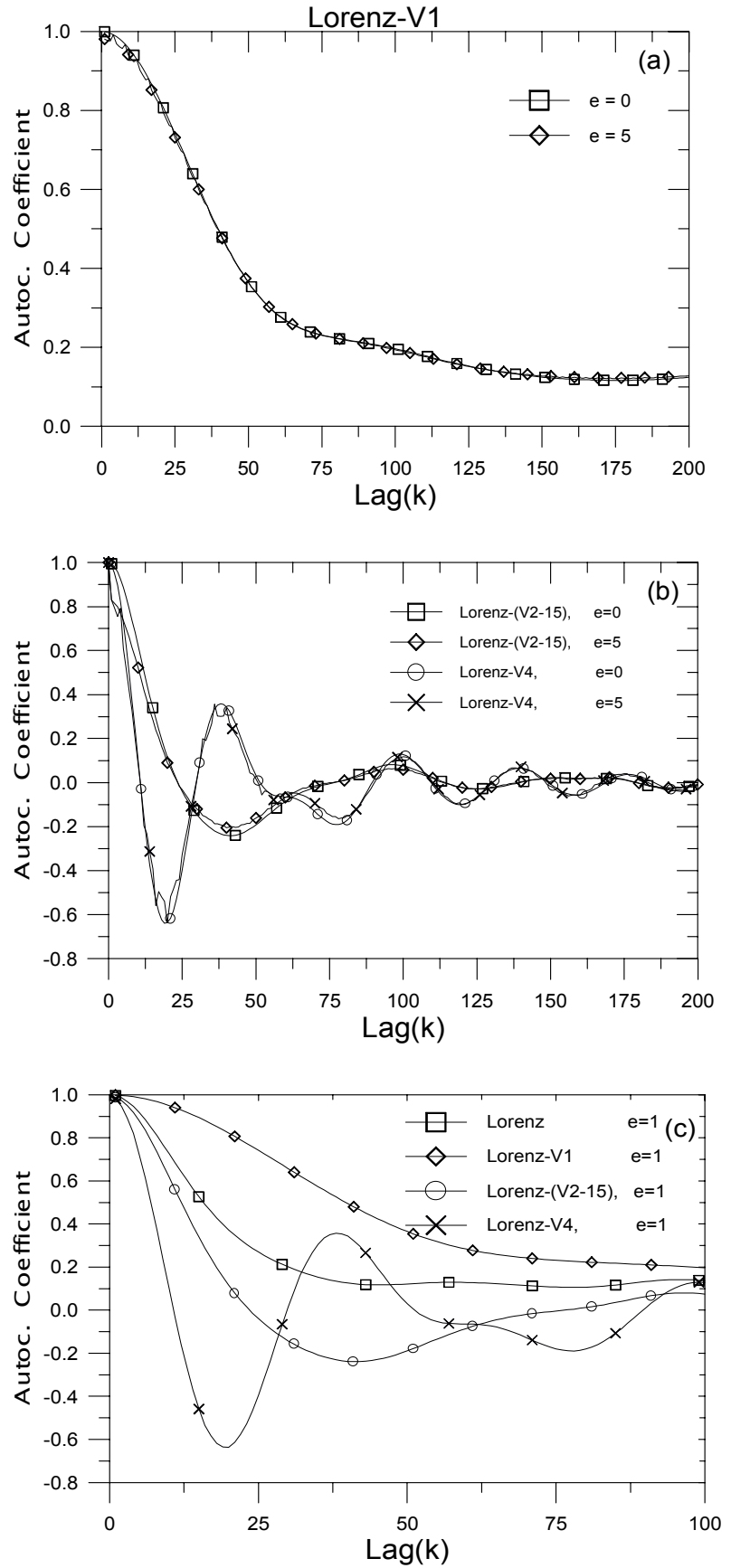

Fig. 14. (a) The autocorrelation coefficient of the $V_{1}$ SVD component of the stochastic $x(t)$ Lorenz component corresponding to the level $e=0.5(7.8 \%)$ of the external white noise perurbation compared to the autocorrelation coefficient of the original signal $(e=0)$. (b) The same as (a) for the $V_{4}, V_{2-15}$ SVD components of the stochastic $x(t)$ Lorenz signal corresponding to the external white noise perturbation level $e=5(39 \%)$. (c) The autocorrelation coefficient of the $x(t)$ stochastic Lorenz signal and its $V_{1}, V_{4}, V_{2-15} \mathrm{SVD}$ component corresponding to the external white noise perturbation level $e=1(7.8 \%)$.

This figure reveals that the perturbation of dynamic system by white noise leaves invariant the nontrivial eigenvalues of
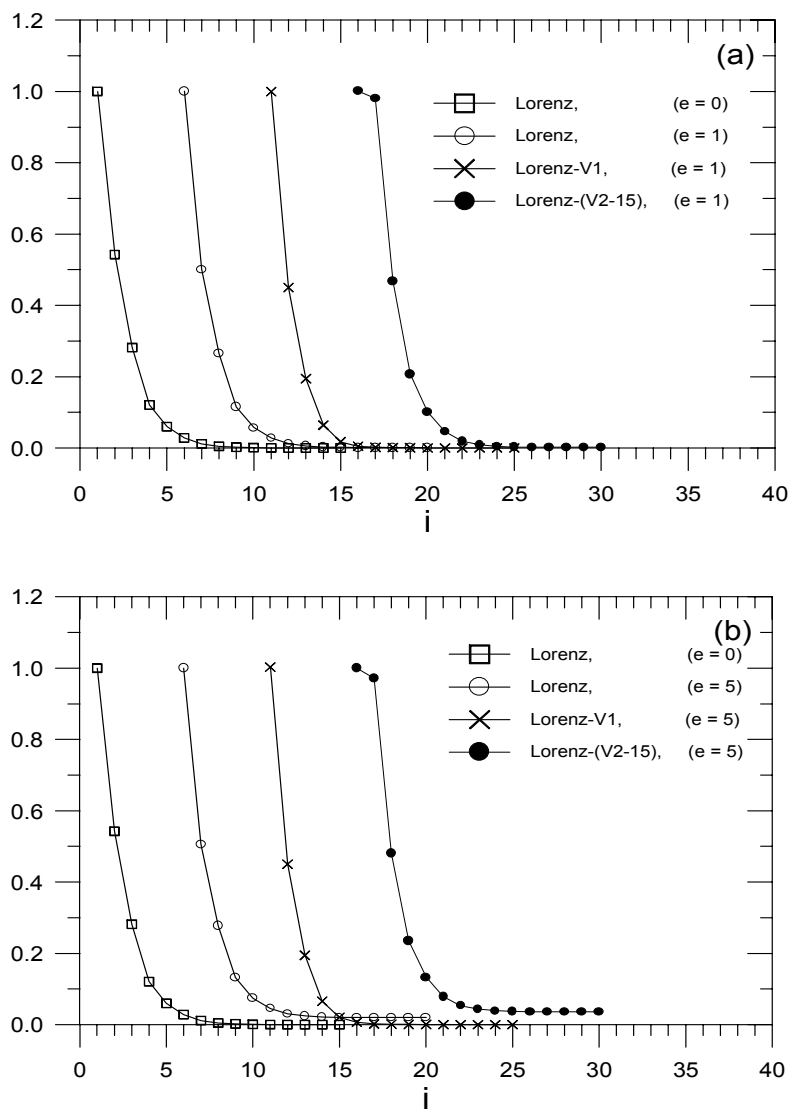

Fig. 15. (a,b) The spectra of the singular values $\sigma_{i}, i=1-25$ corresponding to the purely deterministic $x(t)$ Lorenz signal, the $x(t)$ stochastic Lorenz signal and its $V_{1}, V_{2-15}$ SVD components estimated for the two levels $e=1(7.8 \%)$ and $e=5$ (39\%) of the external white noise perturbation.

the original time series and its SVD components, raising only the noise background. This is in accordance with the theoretical concepts presented in Section 2.4 of this paper.

\subsection{Perturbation by external colored noise}

In this section we apply the SVD analysis to the Lorenz system perturbed by external additive colored noise. The external colored noise is obtained by the equation

$X\left(t_{i}\right)=\sum_{i=1}^{M / 2} C_{k} \cos \left(\omega_{k} t_{i}+\phi_{k}\right), \quad i=1, \ldots, M$

where the phases $\phi_{k}$ are randomly distributed on the interval $[0,2 \pi]$ and $C_{k}$ are constants related to the power spectrum $P\left(\omega_{k}\right)$ by

$C_{k}=\left[P(\omega) \frac{2 \pi}{M} \frac{1}{\Delta}\right]^{1 / 2}$

These random time series have power spectra $P(\omega)$ of the form $\omega^{-\alpha}$ and show low-dimensional chaos, with correlation dimension $D$ related to $\alpha$ through the relation $D=2 /(\alpha-1)$, according to Osborne and Provenzale (1989). 

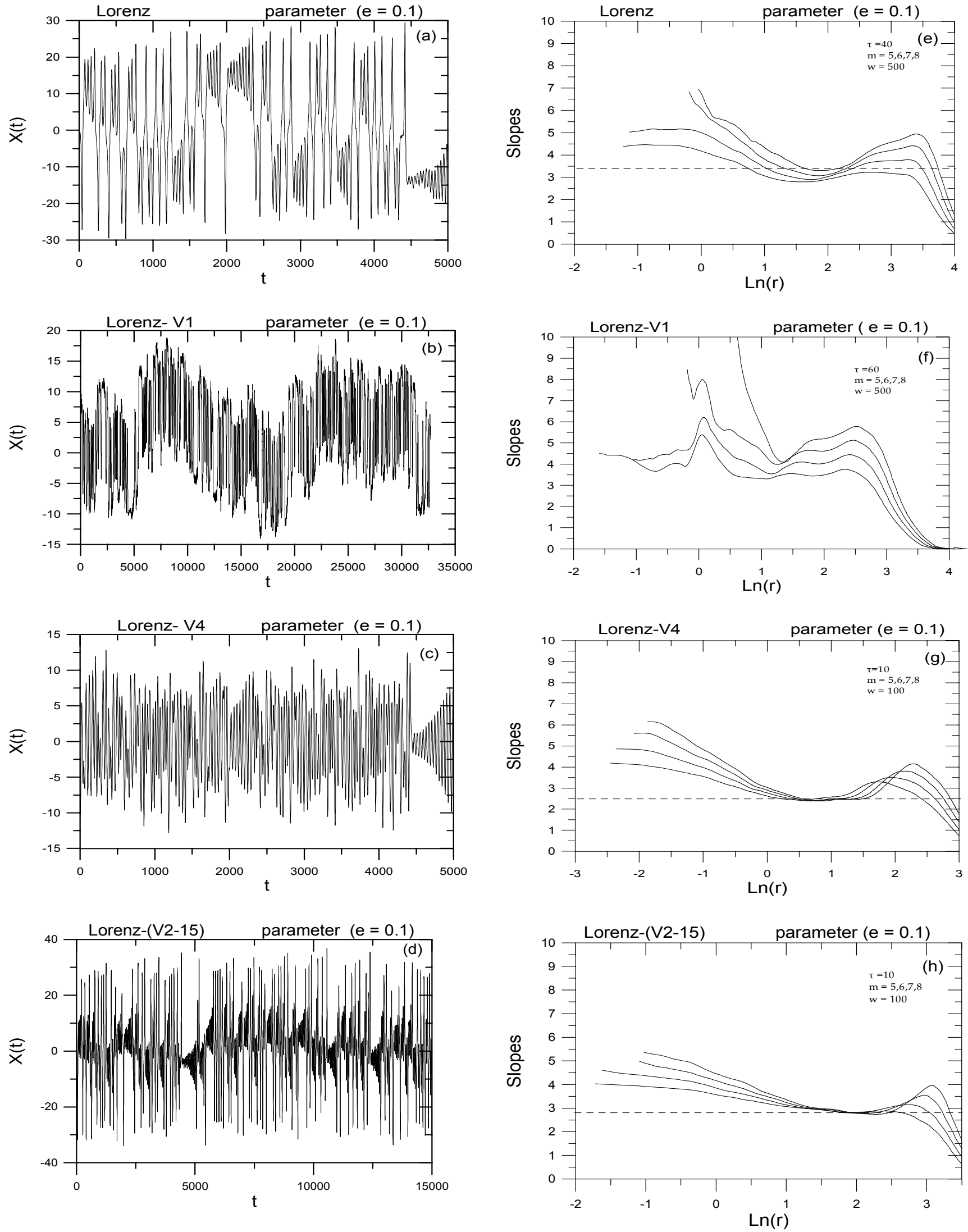

Fig. 16. The same as Fig. 13 but for the level $e=0.1(37 \%)$ of the external colored noise perturbation. 

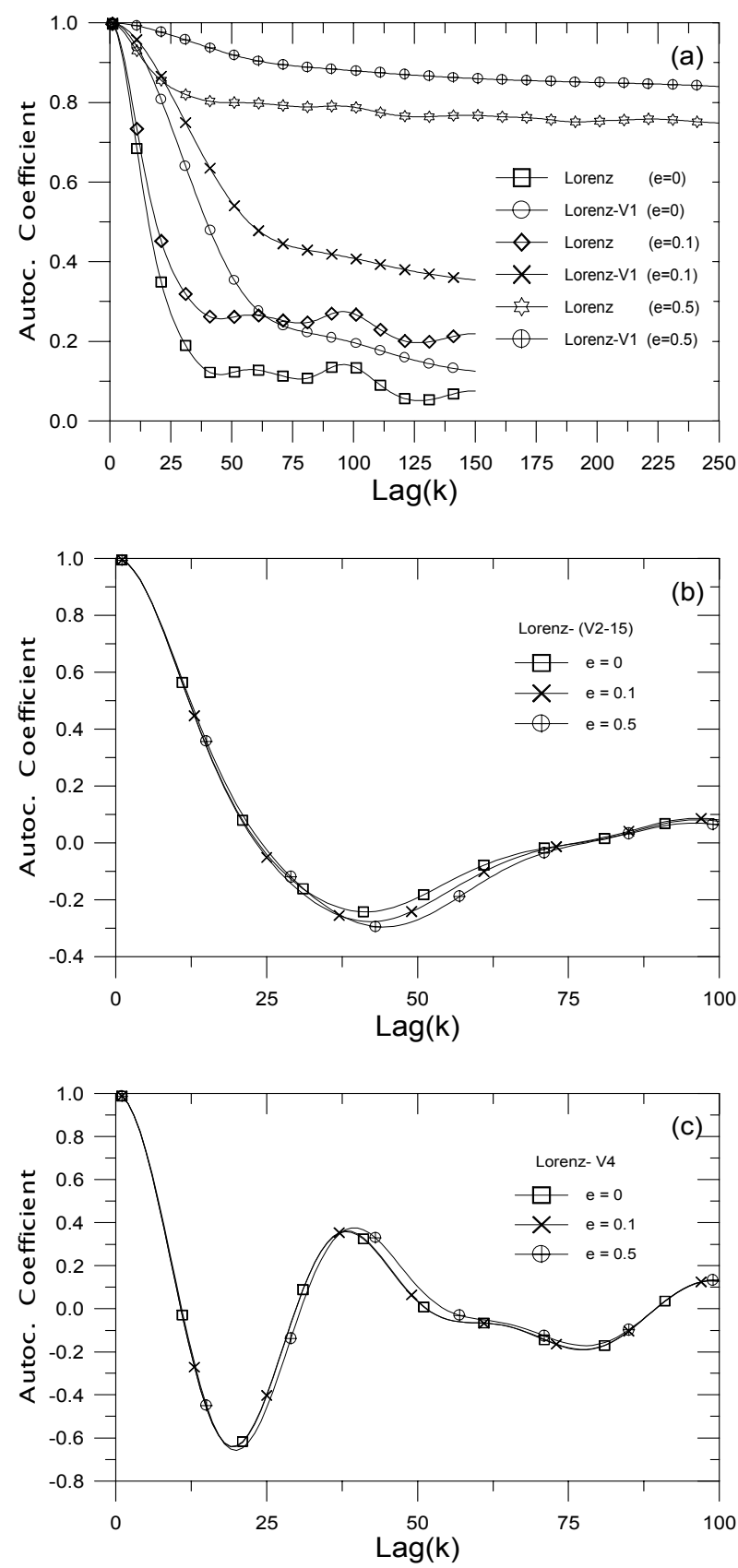

Fig. 17. (a) The autocorrelation coefficient of the deterministic $x(t)$ Lorenz signal, the stochastic $x(t)$ Lorenz signal and its $V_{1}$ SVD component corresponding to the two levels $e=0.1(37 \%)$ and $e=0.5(185 \%)$ of the external additive colored noise perturbation. (b) The autocorrelation coefficients of the $V_{2-15}$ SVD component of the stochastic $x(t)$ Lorenz signal corresponding to the two levels $e=0.1(37 \%)$ and $e=0.5(185 \%)$ of the external additive colored noise perturbation. (c) The same as (b) but for the $V_{4}$ SVD component.

In this section we use two levels of colored noise corresponding to percentage $37 \%(e=0.1)$ and $185 \%(e=0.5)$ according to equation

$x(t)=x_{L}(t)+e x_{\mathrm{cn}}(t)$
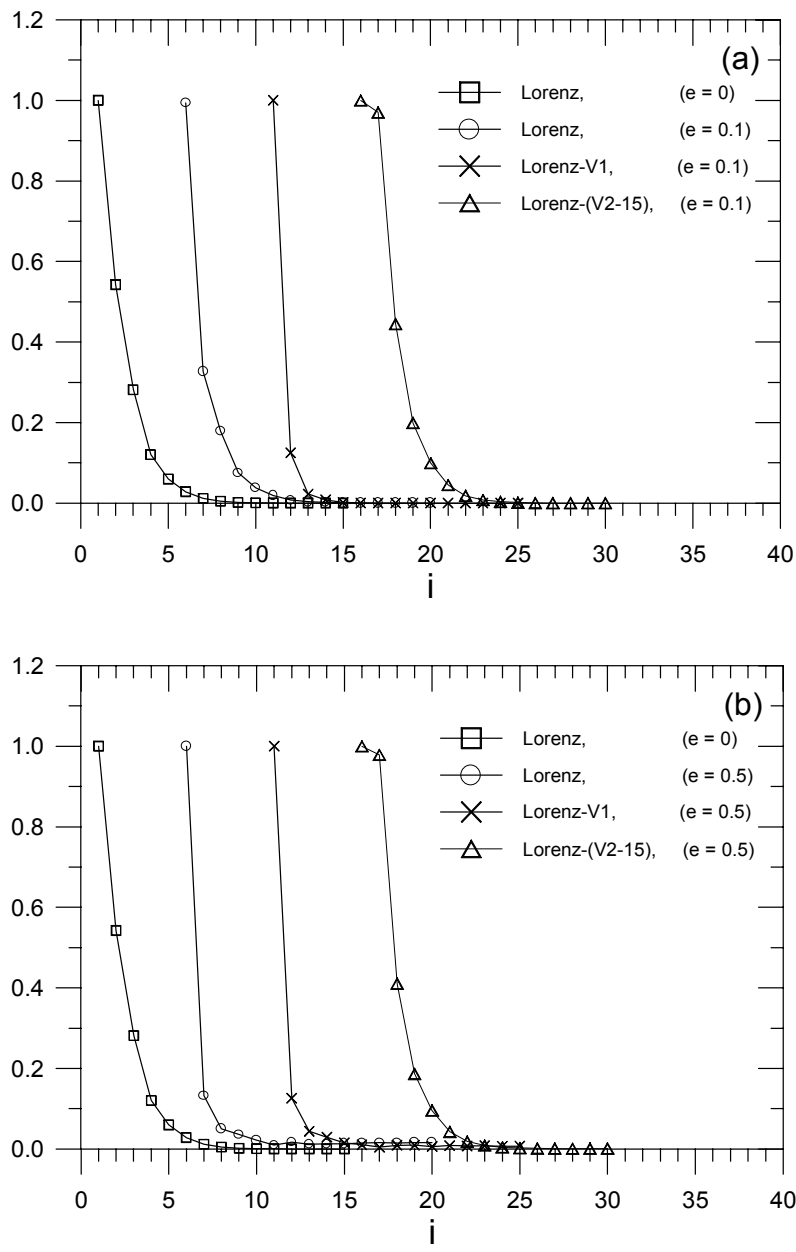

Fig. 18. The same with Fig. 15 but for the colored noise $x(t)$ stochastic Lorenz signal.

where the $x_{\mathrm{cn}}(t)$ time series was obtained to have mean value 38 and standard deviation 47.

Fig. 16a shows the stochastic $x$-Lorenz time series including $37 \%$ of external colored noise. Fig. 16b shows the first SVD component $V_{1}$ of the stochastic signal shown in Fig. 16a. The SVD components $V_{4}$ and $V_{2-15}$ are shown in Figs. 16(c-d). The component $V_{2-15}$ approximates the original time series (shown in Fig. 16a) while the component $V_{1}$ approximates the typical profile of a nonstationary colored noise. The component $V_{4}$ includes noticeable information from the original signal shown in Fig. 16a. Figs. 16(e-h) present the corresponding slopes of the correlation integrals estimated for the time series of Figs. 16(a-d). The slopes shown in the Fig. 16e correspond to the original stochastic signal and the slopes shown in Figs. 16(f-h) correspond to the slopes of the SVD components $V_{1}, V_{4}$ and $V_{2-15}$ of the original Lorenz colored noise stochastic signal. The slopes of the original stochastic signal (Fig. 16e) reveal a small tendency for scaling and saturation of the scaling exponents at the values $D=3-4$ in the range $\Delta \ln r=1-3$ of the distance $r$ in the reconstructed phase space. However, this weak 
Table 3. The same as Table 2 but for the two levels $e=0.1(37 \%), e=0.5(185 \%)$ of the external additive colored noise perturbation.

\begin{tabular}{|c|c|c|c||c|c|c|}
\hline \multicolumn{7}{|c|}{ Lorenz with colored noise } \\
\hline & $e$ & Noise $\%$ & $\sigma_{2} / \sigma_{1}$ & $e$ & Noise $\%$ & $\sigma_{2} / \sigma_{1}$ \\
\hline Lorenz (stochastic) & 0.1 & 37 & 0.33 & 0.5 & 185 & 0.04 \\
\hline$V_{1}$ & 0.1 & 37 & 0.12 & 0.5 & 185 & 0.02 \\
\hline$V_{2-15}$ & 0.1 & 37 & 0.45 & 0.5 & 185 & 0.41 \\
\hline
\end{tabular}

Table 4. The same as Table 3 but for the two levels $e=1(7.8 \%), e=5(39 \%)$ of the external white noise perturbation.

\begin{tabular}{|c|c|c|c||c|c|c|}
\hline \multicolumn{7}{|c|}{ Lorenz with white noise } \\
\hline & $e$ & Noise $\%$ & $\sigma_{2} / \sigma_{1}$ & $e$ & Noise $\%$ & $\sigma_{2} / \sigma_{1}$ \\
\hline Lorenz (stochastic) & 1 & 7.8 & 0.50 & 5 & 39 & 0.50 \\
\hline$V_{1}$ & 1 & 7.8 & 0.45 & 5 & 39 & 0.45 \\
\hline$V_{2-15}$ & 1 & 7.8 & 0.47 & 5 & 39 & 0.47 \\
\hline
\end{tabular}

profile of scaling and saturation is entirely destroyed passing to the $V_{1}$ component, as we can see in Fig. $16 \mathrm{f}$.

A significant profile of scaling and low value saturation of the slopes reappears passing to the next SVD components $V_{4}$ and $V_{2-15}$ as we can see in Figs. 16(g-h). The correlation dimension estimated for $V_{4}$ and $V_{2-15}$ time series was found to be $\sim 2.5$. This value is not much different from the correlation dimensions of the purely deterministic Lorenz system. The above results reveal three significant characters concerning the colored noise:

a) The colored noise causes sensitive raising of the saturation value $D$ of the scaling exponents.

b) The colored noise perturbation is absorbed mainly by the $V_{1}$ SVD component destroying the scaling and low value saturation profile of the slopes corresponding to the $V_{1}$ SVD component.

c) The higher SVD components $\left(V_{4}, V_{2-15}\right)$ absorb much lower percentage of the noise. The correlation dimension of these SVD components is not much different from the correlation dimension of the purely deterministic system.

The above characteristics reveal strong differences between the behavior of the colored and the white noise as we perturb a dynamical system concerning the correlation dimension. The case of the white noise leaves invariant the correlation dimension passing from the original stochastic signal to its SVD components. However, the colored noise leaves invariant only the high SVD components.

In the following we present results concerning the autocorelation coefficient and the singular value spectrum of the Lorenz system perturbed by colored noise. Fig. 17a shows the autocorrelation coefficient estimated for the original $(e=$ $0)$ Lorenz system and for the Lorenz system perturbed by external colored noise corresponding to amplitudes $(e=0.1$, $e=0.5$ ). In the same figure we present the autocorrelation coefficient of the first SVD component $V_{1}$ of the origi- nal stochastic signal. Here it is important to notice the clear difference between the original signal and its $V_{1}$ SVD component concerning the decorrelation time. This characteristic is similar for the colored and white noise. On the other hand, the behavior of the colored noise is different from that of the white noise passing from one level of noise to the next for both cases of the original signal and its $V_{1}$ component. That is, in the case of the colored noise the decorrelation time increases as we increase the amplitude of the external perturbation for both the original signal and its first SVD component $V_{1}$. This is opposite to the behavior of the white noise perturbation as we concluded by using Figs. 12b and 14a. The autocorreletion coefficients for the next SVD components $\left(V_{4}, V_{2-15}\right)$ of the original stochastic signal are shown in Figs. 17(b-c). These figures reveal similar behavior for the colored noise and the white noise (see Fig. 14b), as the decorrelation time of the $V_{4}, V_{2-15}$ SVD components remains invariant passing from weak to strong external perturbation.

Figs. 18(a-b) show the singular values spectrum estimated for the colored noise stochastic Lorenz system and its SVD components $V_{1}, V_{2-15}$. Fig. 18a corresponds to the first level $(e=0.1$ ) of the colored noise perturbation and Fig. $18 \mathrm{~b}$ to the second level $(e=0.5)$. As we can conclude by these figures the colored noise destroys the normal character of the nontrivial eigenvalues for the stochastic signal and its $V_{1}$ SVD component causing the ratio $\sigma_{2} / \sigma_{1}$ of the second to first eigenvalue to increase passing from the purely deterministic to the stochastic signal. However, this effect is not observed for the $V_{2-15}$ SVD component. Table 3 presents the ratio $\sigma_{2} / \sigma_{1}$ estimated for the original Lorenz system, the stochastic (colored noise) Lorenz system and the SVD components of the stochastic Lorenz system. For both levels of perturbation $(e=0.1,0.5)$ the ratio decreases passing from the purely deterministic system to the corresponding stochastic system and the $V_{1}$ component of the stochastic system. In contrast, the ratio $\sigma_{2} / \sigma_{1}$ increases passing from the $V_{1}$ to the $V_{2-15}$ SVD component obtaining almost the value of $\sigma_{2} / \sigma_{1}$ 
estimated for the purely deterministic Lorenz system. The above results reveal that similarly with the correlation dimension and the autocorrelation coefficient the behavior of the colored noise is strong discriminated by the white noise concerning the singular value stectrum. Table 4 is similar to Table 3 corresponding to the case of perturbed Lorenz system by white noise. This table shows that the ratio $\sigma_{2} / \sigma_{1}$ is almost invariant passing from the purely deterministic signal to the corresponding stochastic signal and the SVD components of the second.

\section{SVD analysis and phase portraits}

In this section we study the two-dimensional phase portraits of the AE index system, the stochastic Lorenz system, as well as their SVD components. Figs. 19(a-d) show the two dimensional phase portrait of the $\mathrm{AE}$ index time series and its SVD components $V_{1}, V_{4}, V_{2-10}$. The phase portrait of the original time series (AE index) reveals some kind of structure extended along the axis $X(t), X(t+60)$. The phase portrait of the $V_{1}$ component (shown in Fig. 19b) reveals approximately the same structure to the original time series. However, the Brownian character is stronger in the phase portrait of the $V_{1}$ component. The phase portraits of the next SVD components $V_{4}$ and $V_{2-10}$ (shown in Figs. 19(c-d)) reveal a structure much different than the structure of the original time series (AE index) and its $V_{1}$ component. Now the structure $V_{2-10}$ is extended along the diagonal while the structure of the $V_{4}$ component is more spherical than the structure of the $V_{2-10}$ component or the structures corresponding to the $\mathrm{AE}$ and its $V_{1}$ component.

Figs. 20(a-d) are similar to Figs. 19(a-d) but for the $x$ component of the Lorenz system. As Fig. 20b and Fig. $20 \mathrm{~d}$ reveal, the phase portraits of the $V_{1}, V_{4}$ components are different from the phase portrait of the original signal. The phase portrait of the $V_{2-15}$ component reveals a structure almost similar to the original signal.

Figs. 21(a-d) show the phase portraits of the colored noise $(e=0.1)$ stochastic Lorenz system and its SVD components. Now the structures of the original signal (stochastic Lorenz signal) and its SVD components are weakly changed compared to those shown in Figs. 20(a-d), which correspond to the purely deterministic Lorenz system. In more details the phase portrait of the $V_{2-15}$ component remains invariable passing from the purely deterministic (Fig. 20c) to the stochastic component (shown in Fig. 21c). However, the component $V_{1}$ changes sensibly passing from the purely deterministic to the stochastic signal (see Fig. 20b, 21b).

When the amplitude of the external colored noise perturbation becomes stronger, corresponding to the value $e=0.5$ of the noise parameter, then we can observe some important new characteristics. Now opposite to the previous case of weak perturbation $(e=0.1)$ the colored noise has entirely destroyed the structures of the original stochastic signal and its $V_{1}$ component, as we can conclude by comparing Figs. 20(a-b) and Figs. 22(a-b). However, it leaves invariant the structures of the next components $V_{4}, V_{2-15}$, as we can conclude by comparing Figs. 20(c-d) and Figs. 22(c-d).

Figs. 23(a-d) and Figs. 24(a-d) are similar to the previous Figs. 21(a-d) and Figs. 22(a-d) but they correspond to two different levels of white noise perturbation. When the external white noise perturbation is weak $(7.8 \%$ corresponding to the value $e=1$ of the noise parameter) then the phase portraits of the original stochastic time series (Fig. 23a) and its SVD components (Fig. 23(b-d)) are similar to the corresponding phase portraits of the purely deterministic signal (shown in Figs. 20(a-d)) except for the small trembling caused by the external white noise. However, contrary to the case of the colored noise, when the external white noise becomes strong enough (39\% corresponding to the noise parameter $e=5$ ) the initial structure is covered by the external perturbation as we can see in Figs. 24(a-d). This characteristic is observed both for the initial stochastic signal (see Fig. 24a ) and its SVD components (see Figs. 24(b-d)). The observed difference between the behavior of the white and colored noise perturbation becomes more significant if we note the following: the white noise perturbation related to the noise parameter $\mathrm{e}=5$ corresponds to the percentage of $\sim 39 \%$ of the original signal amplitude. This percentage of white noise is efficient to destroy the original phase space structures for both the original signal and its SVD components. In contrast, the much higher percentage $185 \%$ of the colored noise perturbation corresponding to the value $e=0.5$ of the noise parameter can destroy only the phase structures of the original signal and its $V_{1}$ component (see Figs. 22(a-b)), while it leaves invariant the phase space structures of the next SVD components $V_{4}, V_{2-15}$ (see Fig. 22(c-d)).

Summarizing the above results referred to in the Lorenz system we can conclude that the two dimensional phase portraits of the original signals and their SVD components are also efficient to discriminate between the white and colored noise perturbations. Moreover, even when the colored noise perturbation is much stronger in amplitude than the amplitude of the original signal, the SVD analysis permits one to recapture the original phase space structure by using the SVD components next to $V_{1}$, such as the $V_{2-15}$ SVD component which will be described in the next sections.

By using the above results concerning the stochastic Lorenz system we can conclude that the $V_{2-10}$ SVD components of the $\mathrm{AE}$ index is appropriate for extracting useful information about the underlying dynamical process in the case where the AE index includes strong colored noise. The last supposition about the colored noise component in the $\mathrm{AE}$ index can be supported by the previous results concerning the Lorenz system perturbed by external colored noise. 

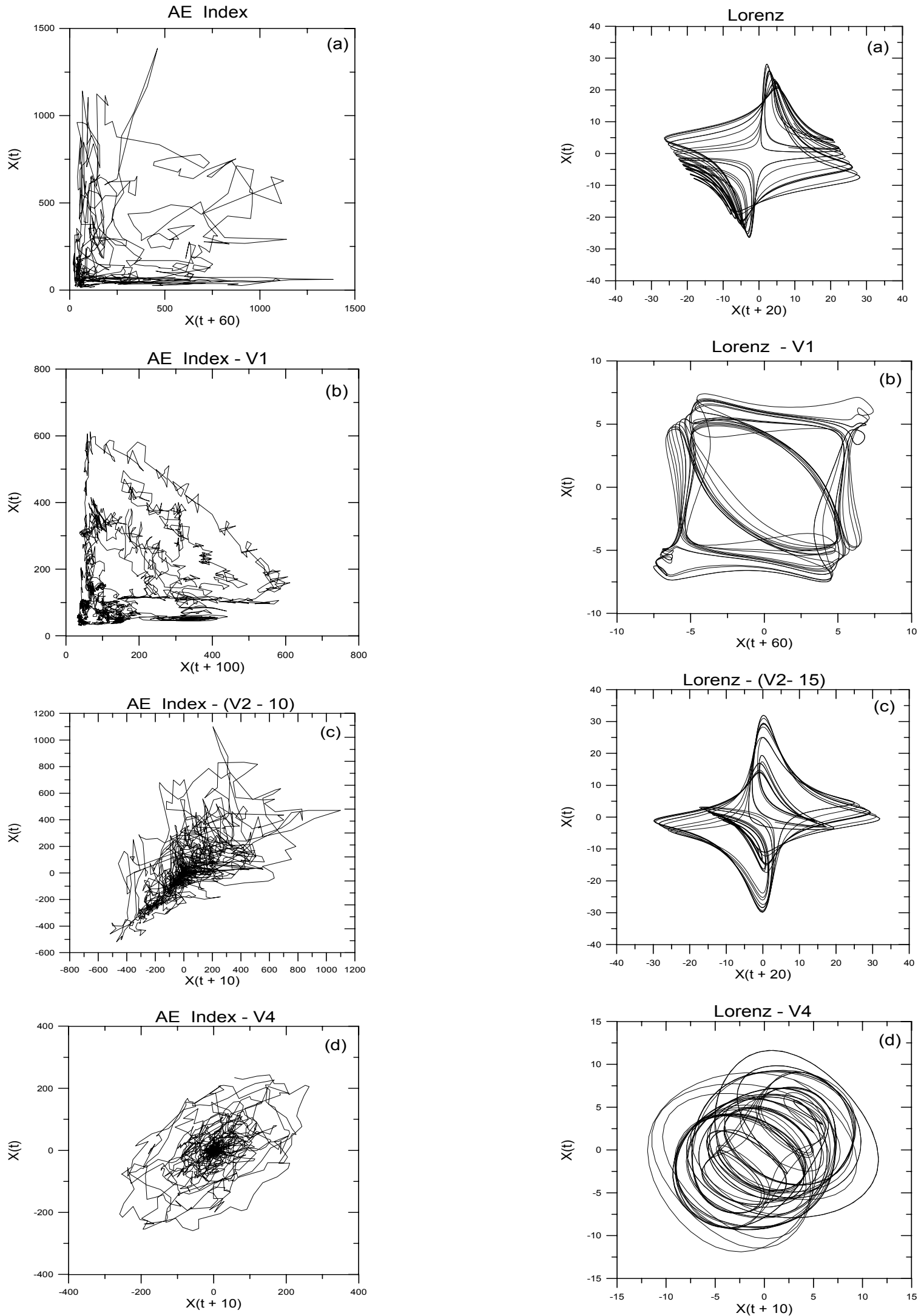

Fig. 19. Two dimensional phase portraits estimated for the $\mathrm{AE}$ index time series and its $V_{1}, V_{4}, V_{2-10}$ SVD reconstructed compo-

Fig. 20. The same as Fig. 19 but for the $x(t)$ Lorenz signal. nents. 

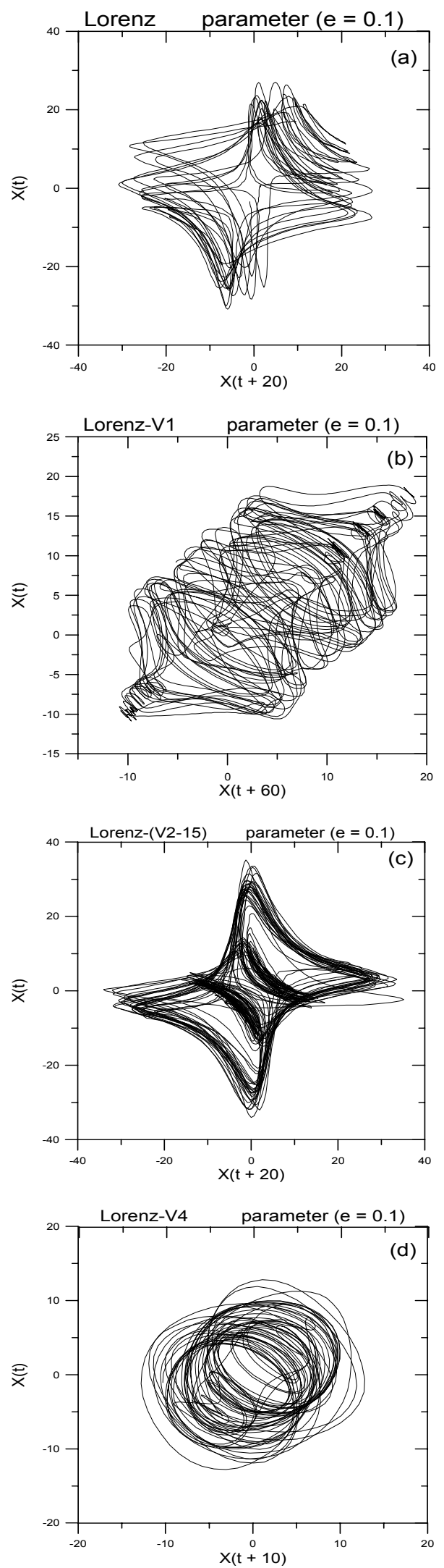

Fig. 21. The same as Fig. 19 but estimated for the $x(t)$ stochastic Lorenz signal corresponding to the level $e=0.1(37 \%)$ of the external colored noise perturbation.
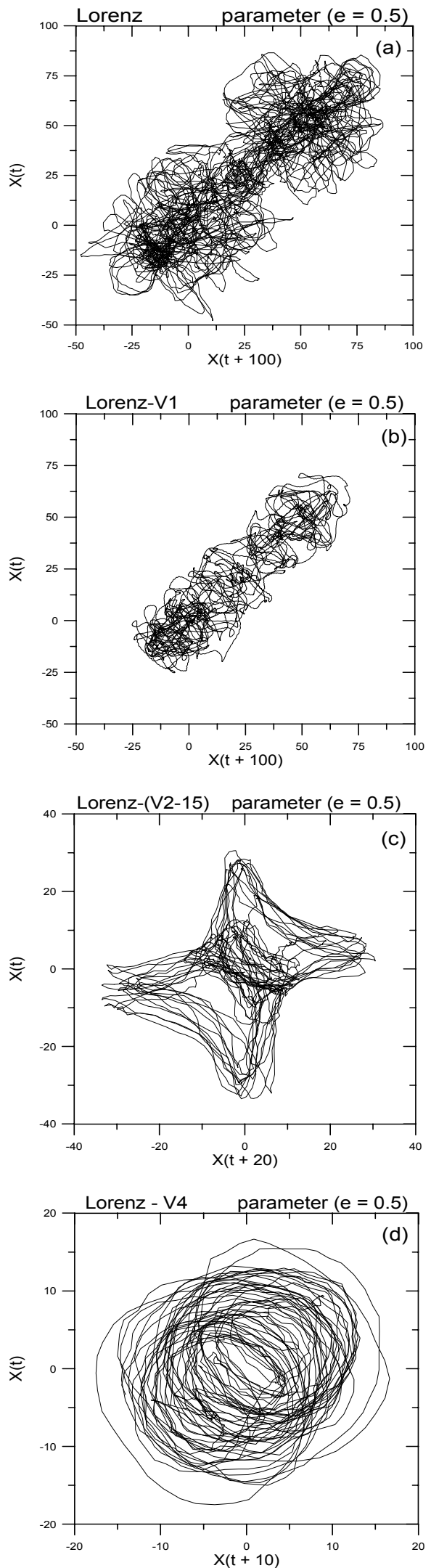

Fig. 22. The same as Fig. 21 corresponding to the level $e=$ $0.5(185 \%)$ of external colored noise perturbation. 

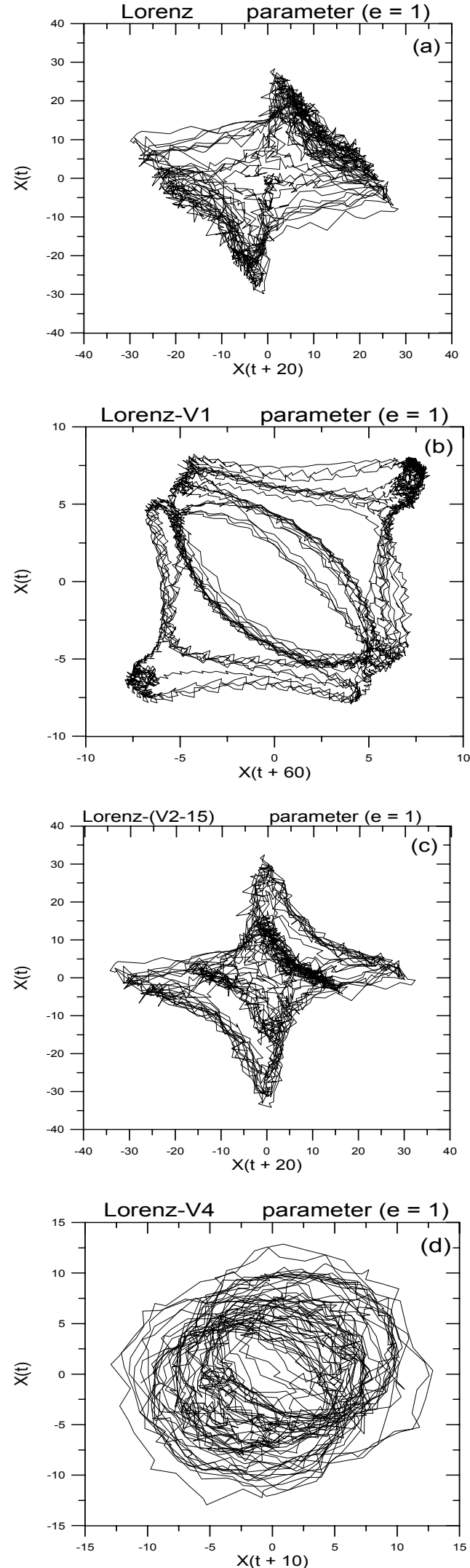

Fig. 23. The same as Fig. 19 but estimated for the $x(t)$ stochastic Lorenz system corresponding to the level $e=1(7.8 \%)$ of the external additive white noise perturbation.
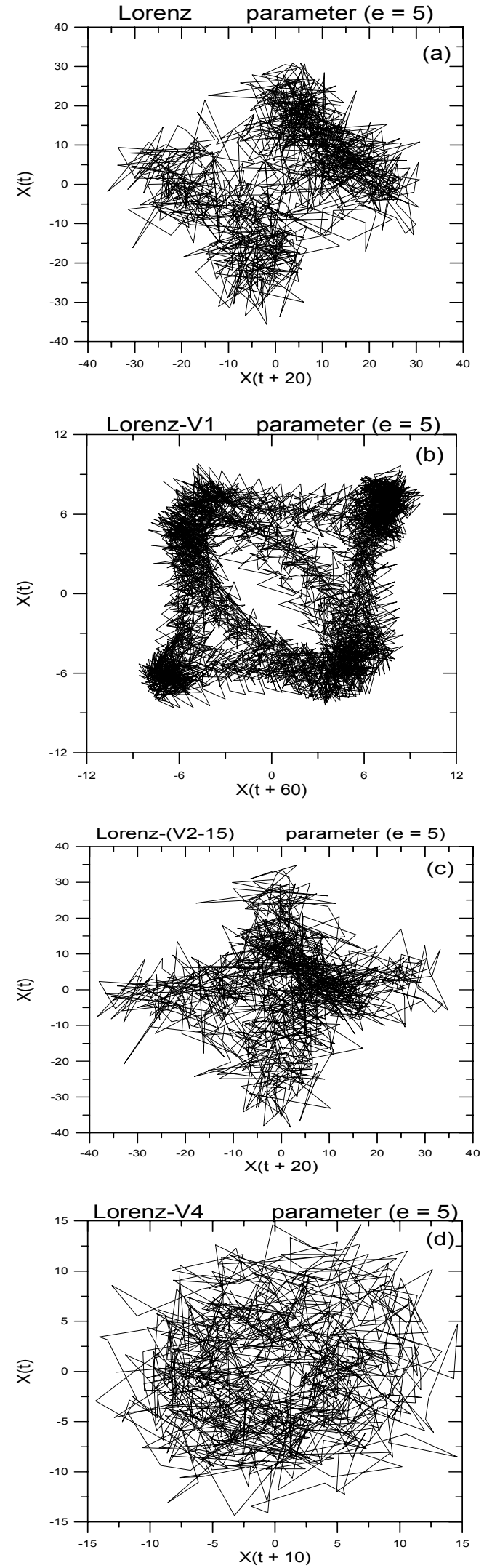

Fig. 24. The same as Fig. 23, but corresponding to the level $e=$ $5(37 \%)$ of the external white noise perturbation. 
Table 5. This shows the cross correlation coefficients of the stochastic $x(t)$ Lorenz signal and its $V_{1}, V_{4}, V_{2-15}$ SVD reconstructed components for two levels $e=1(7.8 \%), e=5(39 \%)$ of external white noise perturbation compared with the corresponding values of the original (deterministic) $x(t)$ Lorenz signal.

\begin{tabular}{|c|c|c|c|c|}
\hline \multicolumn{5}{|c|}{ Lorenz with white noise } \\
\hline \multicolumn{2}{|c|}{} & \multicolumn{3}{|c|}{ Cross Correlation Coefficient } \\
\hline 0 & Noise \% & $V_{1}$ & $V_{2-15}$ & $V_{4}$ \\
\hline 1 & 0.0 & 0.47 & 0.91 & 0.40 \\
\hline 5 & 7.8 & 0.47 & 0.90 & 0.40 \\
\hline
\end{tabular}

Table 6. The same as Table 5 but for the case of white noise perturbation of the Lorenz system.

\begin{tabular}{|c|c|c|c|c|}
\hline \multicolumn{5}{|c|}{ Lorenz with colored noise } \\
\hline \multicolumn{2}{|c|}{} & \multicolumn{3}{|c|}{ Cross Correlation Coefficient } \\
\hline$e$ & Noise $\%$ & $V_{1}$ & $V_{2-15}$ & $V_{4}$ \\
\hline 0.0 & 0.0 & 0.47 & 0.91 & 0.40 \\
\hline 0.1 & 37 & 0.59 & 0.83 & 0.37 \\
\hline 0.5 & 185 & 0.91 & 0.09 & 0.07 \\
\hline
\end{tabular}

\section{Other comparisons of the original signals and their SVD components}

\subsection{Cross correlation of the original signals and their SVD} components

The correlation of the original Lorenz signal (deterministic or stochastic) with its SVD components is shown in Table 5 as a function of the white noise level $(e=0,1,5)$ and in Table 6 as a function of the colored noise level $(e=0,0.1,0.5)$. For example, the column $V_{1}$ in Table 5 presents the cross correlation of the original Lorenz signal and its $V_{1}$ component as a function of the white noise level. The first column of every table shows the percentage of the noise corresponding to the noise parameter.

Concerning the white noise we notice that the cross correlation of the original Lorenz signal and its SVD components $\left(V_{1}, V_{4}, V_{2-15}\right)$ is approximately independent of the external perturbation and equal to the original cross correlation estimated for the value $e=0$ of the noise parameter (case of zero perturbation).

It is important to note in Table 5 that the cross correlation of the original signals and their $V_{1}$ and $V_{4}$ components are equal to the value $\sim 0.4-0.5$. Contrary, the cross correlation value of the original signals and their $V_{2-15}$ SVD component is much higher and equal to $\sim 0.9$. This result is expected as the component $V_{2-15}$ approximates the original signal much better than the $V_{1}$ or $V_{4}$ components, according to the theoretical concepts presented in Section 2.

Concerning the colored noise perturbation we observe different behavior of the cross correlations. As we can see in Table 6 the cross correlation of the original signal with its
Table 7. The same as Table 5 corresponding to the magnitudes of the standard deviation.

\begin{tabular}{|c|c|c|c|c|c|}
\hline \multicolumn{7}{|c|}{ Lorenz with white noise } \\
\hline \multicolumn{2}{|c|}{} & \multicolumn{5}{|c|}{ Standard deviation } \\
\hline 0 & Noise \% & Original & $V_{1}$ & $V_{2-15}$ & $V_{4}$ \\
\hline 0 & 0.0 & 12.69 & 5.29 & 11.17 & 5.19 \\
\hline 5 & 7.8 & 12.73 & 5.32 & 11.24 & 5.21 \\
\hline 5 & 39 & 13.63 & 5.40 & 12.20 & 5.65 \\
\hline
\end{tabular}

Table 8. The same as Table 6 but for the standard deviation.

\begin{tabular}{|c|c|c|c|c|c|}
\hline \multicolumn{7}{|c|}{ Lorenz with colored noise } \\
\hline \multicolumn{2}{|c|}{} & \multicolumn{5}{|c|}{ Standard deviation } \\
\hline 0.0 & 0.0 & 12.69 & 5.29 & 11.17 & 5.19 \\
\hline 0.1 & 37 & 13.78 & 7.62 & 11.11 & 5.31 \\
\hline 0.5 & 185 & 27.47 & 24.06 & 11.29 & 6.91 \\
\hline
\end{tabular}

$V_{1}$ component increases as we increase the amplitude of the external perturbation. In contrast to this, the behavior of the cross correlations corresponding to the SVD components $V_{4}$ and $V_{2-15}$ decreases as we increase the amplitude of the external perturbation for both cases of $V_{4}$ and $V_{2-15}$ SVD components.

Comparing the previous results about the dependence of the correlation of the initial signals with the SVD components upon the level of the external noise, we can conclude the following: the white noise perturbs symmetrically all of the SVD components of the original signal, while the colored noise perturbs mainly the first SVD component $V_{1}$ and leaves invariant the next components.

\subsection{Standard deviation of the original signals and their SVD components}

The standard deviation, defined as the square root of the variance, was computed for the original signal and its SVD reconstructed components in the case of the Lorenz system perturbed by external white and colored noise. Table 7 shows the standard deviations in the case of white noise as a function of the noise level. As we increase the level of noise the standard deviation does not increase significantly in the case of the original signal as well as in the cases of its SVD components $V_{1}, V_{4}, V_{2-15}$. Contrary, in the case of the colored noise (Table 8 ), the standard deviation of the original signal and its first SVD component $V_{1}$ increases significantly as we increase the level of noise while it remains invariable, especially for the component $V_{2-15}$. In the case of the $V_{4}$ component we notice a very slow increase which becomes $\sim 2$ units for $185 \%$ of noise. For $37 \%$ of colored noise the standard deviation of the $V_{4}$ and $V_{2-15}$ SVD components remain invariable and equal to the standard deviation of the purely deterministic signal. Concluding, we have shown in this section that the study of cross correlations and standard 
deviations indicate clear differences between white and colored noises. That is, while the white noise can be manifested symmetrically in all the SVD components, the colored noise perturbs only the first SVD component. The above results are similar with the results included in previous sections.

\section{Summary and discussion}

In this paper we applied the SVD analysis to the magnetospheric AE index and the Lorenz system. In the case of the Lorenz system we studied it in three different stages: When the system is independent from external noise, as well as when it is perturbed by external white or colored noise. The results of the above analysis are very interesting for the physical understanding of the magnetospheric dynamics. In addition, the results of this paper reveal new possibilities of the SVD analysis improving the algorithm of chaotic analysis of experimental time series. Before we discuss the physical implications of this paper, we summarize the crucial points of the previous sections.

\subsection{The AE index system}

The SVD method used for substituting the original trajectory matrix $\mathbf{X}$ by the $\mathbf{X C}$ trajectory matrix improves effectively the scaling and the saturation profile of the slopes. The slopes estimated by using the $\mathbf{X}$ and $\mathbf{X C}$ trajectory matrices (shown in Figs. 2(a-b)) showed clearly the improvement of the scaling and low-dimensional profile of the slopes after the application of the SVD transformation on the trajectory matrix.

The first SVD reconstructed component $V_{1}$ of the AE index does not reveal low-dimensionality, although it corresponds to the eigenvector $\boldsymbol{c}_{1}$ and the eigenvalue $\sigma_{1}$, which theoretically must include the highest deterministic component of the signal. Moreover, the slopes of the $V_{1}$ component indicate that the $V_{1}$ time series is a high dimensional stochastic signal with long decorrelation time because no significant discrimination with its nonlinear surrogate data is possible. On the other hand, the next SVD components $V_{4}, V_{2-10}$ of the AE index clearly reveal low-dimensional and nonlinear character. The discrimination with nonlinear surrogate data is now effective.

Concerning the singular value spectrum of the AE index we observed the first value $\sigma_{1}$ to be much larger than the next singular values which are pressed to the noise floor. This picture is invariable passing to the first SVD component $V_{1}$, but changes drastically going to the next SVD components. In particular, for the $V_{2-10}$ SVD component, we observed a spectrum of normal singular values which includes a small number $(\sim 7)$ of nontrivial eigenvectors. This number of nontrivial eigenvalues and eigenvectors above the noise background indicates $\sim 7$ dynamical degrees of freedom of the underlying dynamics. This result is in agreement with the correlation dimension $D \cong 2.5$ given that the number of degrees of freedom $(d)$ satisfies the relation
$D_{\text {int }} \leq d \leq 2 D_{\text {int }}+1$, where $D_{\text {int }}$ corresponds to the first integer which is greater than the fractal correlation dimension. For the AE index this relation indicates $d=3-7$ possible degrees of freedom.

\subsection{The Lorenz system}

7.2.1 Purely deterministic low-dimensional dynamical system (Lorenz system)

The correlation dimensions remain invariable passing from the original signal to its SVD components, while the nonlinear surrogate data are clearly discriminated from the original signal, the Lorenz signal and its $V_{1}, V_{4}, V_{2-10}$ SVD components. The singular value spectrum $\left\{\sigma_{i}\right\}$ is also invariable passing from the original Lorenz signal to its SVD components. The phase portrait of the $V_{2-15}$ SVD component is almost similar to the phase portrait of the original signal, while the phase portraits of the independent SVD component $\left(V_{1}, V_{4} \ldots\right)$ are dissimilar to that of the original signal.

\subsubsection{Low-dimensional stochastic dynamics (the case of white noise)}

The study of the stochastic Lorenz system showed that the perturbation of a dynamical system by external white noise leaves invariant the physical characteristics of the system causing only secondary changes. Summarizing the results about the white noise perturbation at a dynamical system we have observed the following:

In the case of white noise perturbation the scaling and saturation profile of the slopes are conserved invariable for a narrow range of distance $r$, but they are destroyed for small values of $r$. This character was observed to be real for the original (purely deterministic system), the white noise stochastic system and their SVD components. That is, the correlation dimension is almost the same for the original purely deterministic signal, the stochastic signal caused by the white noise perturbations, and the SVD reconstructed components of the stochastic time series. The singular value spectrum $\left\{\sigma_{i}\right\}$ is also invariable passing from the original $(e=0)$ to the stochastic $(e=1,5)$ system and from them to the SVD components of the stochastic time series.

The decorrelation time was also found to be invariable passing from the original purely deterministic time series and its SVD components to the corresponding stochastic signals and their SVD components. That is, the white noise perturbation does not change the decorrelation times of the original signal and those of its SVD components. The phase portraits of the white noise stochastic Lorenz system (case of white noise) and its SVD components are invariant for weak white noise perturbation and change drastically for strong white noise perturbation. The same happens for the cross correlations and standard deviations as they remain invariable passing from the purely deterministic Lorenz system to stochastic (after the application of a white noise perturbation) system and its SVD components. Concluding, we can support the 

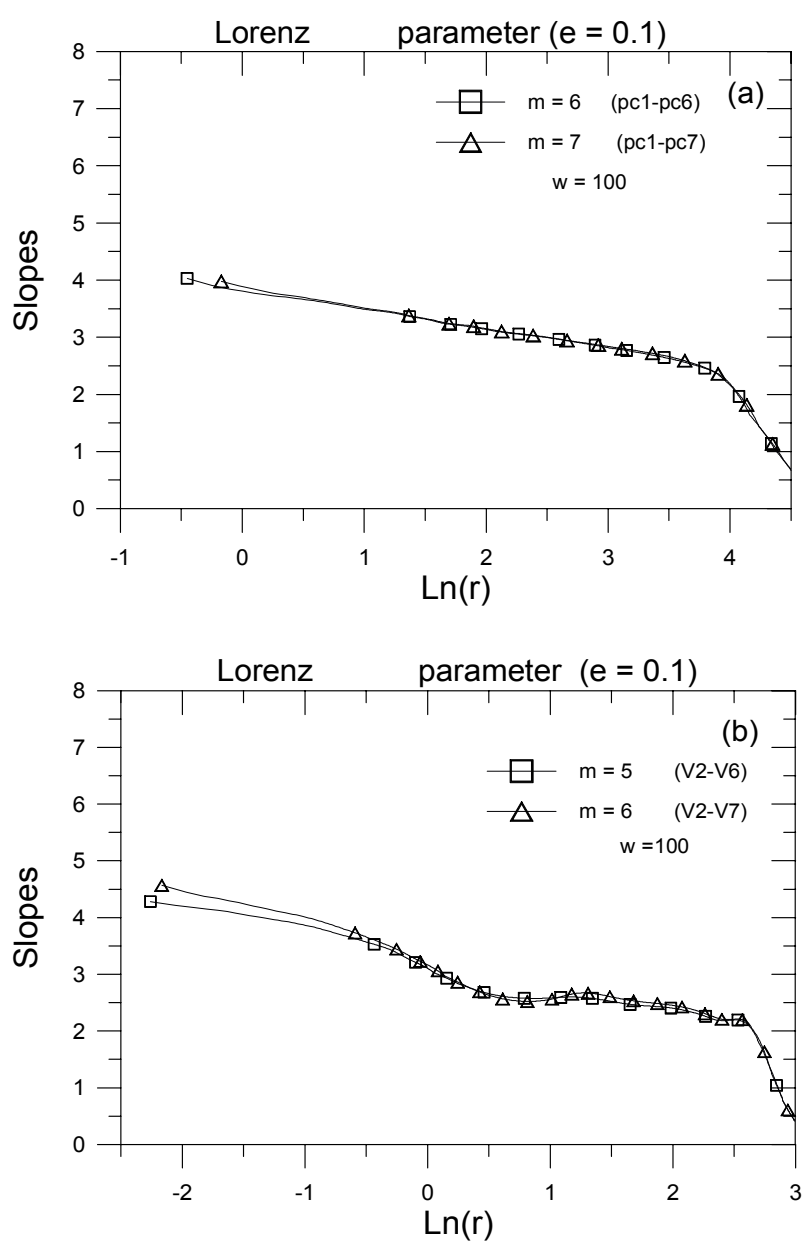

Fig. 25. (a) The slopes of the correlation integrals estimated for the Lorenz colored noise stochastic signal $(e=0.1)$ for embed$\operatorname{ding} m=6,7$. The trajectories were constructed to correspond to the first six and seven columns respectively of the SVD transformed trajectory matrix XC. (b) The same as (a) corresponding to 5-dimensional and 6-dimensional trajectories constructed by using the $V_{2}-V_{6}$ and $V_{2}-V_{7}$ SVD components of the original stochastic signal respectively.

concept that generally the white noise perturbation does not destroy drastically the physical characteristics of the original dynamical system and it is manifested symmetrically on the SVD reconstructed components of the original signal.

\subsubsection{Low-dimension stochastic dynamics (the case of col- ored noise)}

The behavior of a stochastic signal caused by colored noise perturbation upon the SVD analysis is much different from the case of white noise stochasticity or the case of a purely deterministic signal. The crucial points of differentiation are the behavior of the first SVD component $V_{1}$, the first singular value $\left(\sigma_{1}\right)$, the decorrelation times, the cross correlations and the standard deviation of the stochastic signal and its $V_{1}$ component. Summarizing, we have observed the following characteristics:
The decorrelation time increases drastically passing from the original deterministic signal to the corresponding stochastic signal. The increment of the decorrelation time becomes larger for larger amplitudes of perturbation. The same phenomenon was observed for the $V_{1}$ component. That is, the decorrelation time increases passing from the $V_{1}$ component of the original (deterministic) signal to the $V_{1}$ component of the corresponding stochastic signal. The increment is also larger for larger amplitudes of perturbation. Moreover, the first SVD component $V_{1}$ of the colored noise stochastic signal does not reveal any scaling or low value saturation profile. In addition, it is not possible to discriminate the $V_{1}$ component of the stochastic signal from its surrogate data. This behavior indicates that the $V_{1}$ component absorbs the main part of the external colored noise. In contrast, the next SVD components reveal efficient scaling and saturation profiles corresponding to the low-dimensional characteristics of the original deterministic signal.

As for the stochastic system (case of colored noise) and its $V_{1}$ component, the first singular value $\sigma_{1}$ of the singular values spectrum $\left\{\sigma_{i}\right\}$ was observed to be much higher than the next singular values which were pressed down to the noise floor. This characteristic becomes stronger as the amplitude of the colored noise perturbation increases. However, the singular value spectrum becomes normal and is similar to the spectrum of the original deterministic signal passing to the next SVD components of the corresponding stochastic signal.

The scaling and the low-dimensional profile of the slopes of the Lorenz signal perturbed by a colored noise (shown in Fig. 16a) can be improved when we apply the SVD filter. Fig. 25a shows the slopes of the correlation integrals for embedding $m=6,7$ estimated for the colored noise stochastic signal $(e=0.1)$ by using the first six and seven columns of the transform trajectory matrix $\mathbf{X C}$ of the SVD analysis. Comparing Fig. 16e which corresponds to the slopes estimated by the original trajectory matrix $\mathbf{X}$ of the Lorenz stochastic signal, with Fig. 25a which corresponds to the SVD filter signal, we observe significant improvement concerning the scaling and low-dimension profile. However, the improvement is even stronger when we use the $V_{2}-V_{6}$ and $V_{2}-V_{7}$ SVD components for the $m=6$ and $m=7$ embedding. Fig. 25b shows the slopes estimated for $m=6$ and $m=7$ embedding by using the $V_{i}$ components of the stochastic signal as columns of the trajectory matrix. The index takes values $i=2-6$ and $i=2-7$ in the $m=6$ and $m=7$ embedding space respectively. Now the scaling and saturation profile is even better, especially in the range $\Delta \ln r=0.5-2.5$. The saturation value $D \approx 2.5$ of the scaling exponent $\left(d_{m}\right)$ is not much different from the correlation dimension of the original deterministic signal. In contrast to the case of the white noise, the perturbation of the dynamical system by a strong component of colored noise leaves invariant the phase portraits of the $V_{2-15}$ SVD reconstructed components, as well as the phase portraits of the next components $V_{i},(i \geq 2)$. The phase portraits of the stochastic signal and its $V_{1}$ SVD component are changed drastically passing 
from the purely deterministic to the stochastic system. We have observed similar results for the standard deviations and the cross correlations. That is, the standard deviation of the higher SVD component $\left(V_{2-15}, V_{4}\right)$ remains invariant as we increase the amplitude of the colored noise perturbation, but increases drastically for the original stochastic signal and its $V_{1}$ SVD component. The cross correlations of the original stochastic signal and its $V_{2-15}$ SVD component decrease as we increase the colored noise perturbation while the cross correlations of the original stochastic signals and its $V_{1}$ components increase. This behavior reveals that the colored noise perturbs only the $V_{1}$ component. Concluding, we can support the concept that the colored noise perturbation is manifested in the original stochastic signal only through its first $\left(V_{1}\right)$ SVD reconstructed component, while the higher SVD components remain unperturbed and conserve the characteristics of the original purely deterministic system.

\subsection{Comparison of the AE index and the Lorenz system}

The results of Sections 4-6 permit us to conclude significant similarities between the magnetosperic $\mathrm{AE}$ index and the Lorenz stochastic system corresponding to the case of the color noise perturbation. In more detail we have shown the following:

The scaling and low value saturation profile of the AE index are improved sensibly by the SVD filtering. The same happens with the colored noise stochastic Lorenz signal.

The first SVD component $V_{1}$ of the AE index does not reveal low-dimensional profile or efficient possibility for discrimination between the $V_{1}$ component and its surrogate data. The same was observed for the case of the colored noise stochastic Lorenz signal but not for the case of the white noise stochastic Lorenz signal. In contrast to the component $V_{1}$, the next SVD components of the AE index $V_{2-10}, V_{4}$ reveal low-dimensional and nonlinear profile. The same characteristic was observed for the stochastic Lorenz system corresponding to the external colored noise perturbation.

The singular value spectrum of the $\mathrm{AE}$ index reveals strong asymmetry between the values of the first eigenvalue $\left(\sigma_{1}\right)$ and the values of the next eigenvalues $\left\{\sigma_{i}\right\},(i \geq 2)$, which are pressed to the noise floor. The same was observed for the colored noise Lorenz stochastic signal but not for the white noise Lorenz stochastic signal.

The previous result concerning the first $\left(\sigma_{1}\right)$ and the next singular values $\left(\sigma_{i}, i \geq 2\right)$ of the $\mathrm{AE}$ index and the colored noise stochastic signals are conserved effectively passing to the $V_{1}$ SVD component of both signals, the AE index time series and the colored noise stochastic Lorenz system.

The singular value spectrum estimated for the next to $V_{1}$ SVD components of the AE index and for the colored noise Lorenz stochastic signal shows a normal profile, revealing a low number of nontrivial eigenvalues above the noise floor.

In addition, concerning the colored noise stochastic signal (stochastic Lorenz system), the number of nontrivial eigenvalues above the noise floor, estimated for the SVD components $V_{2-15}, V_{4}$, is approximately similar to the number
Table 9. This shows the cross correlation coefficient of the AE index time series and its $V_{1}, V_{4}, V_{2-15}$ SVD components.

\begin{tabular}{|c|c|c|}
\hline \multicolumn{3}{|c|}{ AE Index } \\
\hline \multicolumn{2}{|c|}{ Cross Correlation Coefficient } \\
\hline$V_{1}$ & $V_{2-15}$ & $V_{4}$ \\
\hline 0.63 & 0.72 & 0.34 \\
\hline
\end{tabular}

of nontrivial eigenvalues of the original deterministic Lorenz signal. Similar results were found for the phase portraits and the standard deviations of the colored noise Lorenz system. That is, the phase portraits and the standard deviations of the $V_{2-15}$ and $V_{4}$ SVD components of the colored stochastic signals remain invariant passing from the purely deterministic to the colored noise stochastic Lorenz system, while they change drastically for the stochastic signal and its $V_{1}$ SVD component.

The autocorrelation coefficient of the colored noise stochastic signal shown in Figs. 17(a-c) reveals two different processes: one process with a short decorrelation time corresponding to the deterministic component of the signal and the other process with long decorrelation time corresponding to the colored noise component. The AE index also reveals two independent rates of decorrelation (see Fig. 1c): one rate which is fast and corresponds to low-dimensional dynamics and the other rate which is slow and corresponds to the external colored noise perturbation. The existence of an external high-dimensional colored noise component in the AE index system can be concluded by the similarity which was observed between the $\mathrm{AE}$ index and the colored noise Lorenz system concerning the slopes of the correlation integral signal and the singular value spectrum estimated for the original signals and its SVD reconstructed components. Accepting the hypothesis of the external colored noise component, we can conclude the low-dimensional and nonlinear character of the deterministic component of the $\mathrm{AE}$ index by using the results concerning the correlation dimension and the singular value spectrum. The results concerning the cross correlation, the phase portraits and standard deviation of the higher SVD components $\left(V_{2-10}, V_{4}\right)$ estimated for the colored noise stochastic system in contrast to the $V_{1}$ component conserve the dynamical characteristics of the original purely deterministic low-dimensional system. This strengthens the hypothesis of external colored noise in the AE index system.

In order to estimate in the first approximation the percentage of the external colored noise to the AE index, we estimated the correlation of the AE index time series and its SVD components shown in Table 9. Assuming that the $V_{1}$ component absorbs the main part of the external colored noise component, according to the previous results of this paper, we can conclude $\sim 40 \%$ of external colored noise perturbation of the AE index. This value was concluded by comparing the cross correlation of the $\mathrm{AE}$ index and its $V_{1}$ component shown in Table 9 and the corresponding cross correlation values of the original colored noise stochastic signal and its $V_{1}$ 
component shown in the column $\left(V_{1}\right)$ of the Table 6.

According to the extended analysis presented in our previous paper (Pavlos et al., 1999c ) the supposed colored noise external perturbation of the AE index system must be connected with the solar wind and the earth's magnetosphere interaction. According to our analysis, the solar wind, which causes an external perturbation to the magnetospheric system, must be a high-dimensional colored signal. On the other hand, the previous results permit us to conclude, in addition, that the low-dimensional profile shown by the SVD filter AE index signal and its SVD reconstructed components $V_{2-10}, V_{4}$, strongly support the supposition of an underlying low-dimensional internal magnetospheric process. This process must be nonlinear as it can be supported by the method of surrogate data applied to the SVD reconstructed components of the AE index $V_{2-10}, V_{4}$. The chaotic character of this process is indicated by the fractal dimension $D \approx 2.5$ estimated in this paper by using the SVD analysis as well as by the dynamical characteristics studied in our previous paper (Pavlos et al., 1999b).

Finally, the results of this paper strongly support the theoretical concept that the AE index includes two different physical processes. One process which is low-dimensional and chaotic, corresponding to the internal magnetospheric dynamics, and the other process which is stochastic and strongly colored in its power spectrum and cannot be discriminated by a stochastic linear and high-dimensional process, as it was indicated by studying the first SVD component $V_{1}$ of the AE index and its surrogate data. Therefore, the modeling of these two magnetospheric phenomena remains an open problem. The first phenomenon is related with the low-dimensional chaotic behavior and is purely magnetospheric and the other phenomenon is a rather linear stochastic one and is driven externally to the magnetosperic dynamics as a colored noise perturbation. Moreover, the study of stochastic dynamical systems presented in this paper must be extended for cases including dynamical noises. The external colored noise perturbation of the magnetospheric system must be related to its coupling with the solar wind system, since the solar wind is the main source of dynamical perturbation of the magnetosphere. If this hypothesis is correct then the solar wind input of the magnetosphere must behave as a high-dimensional colored noise. From this point of view, in order to test the above hypothesis concerning the colored noise, the extended chaotic analysis used in this paper must be applied to the solar wind system.

Acknowledgements. We thank Prof. A.G. Rigas for having kindly read our manuscript and for making constructive comments. We thank S. Houmas, N. Stamatopoulos and K. Karadamoglou for their computer support.

\section{References}

Argyris, J., Andreadis, I., Pavlos, G., and Athanasiou, M., On the influence of noise on the Largest Lyapunov exponent and on the geometric structure of attractors, Chaos, Solitons \& Fractals, 9, 947-958, 1998.

Broomhead, D. S. and King, G. P., Extracting qualitive dynamics from experimental data, Physica D, 20, 217-236, 1986.

Baker, D. N., Klimas, A. J., McPherron, R. L., and Bucher, J., The evolution from weak to strong geomagnetic activity: an interpretation in terms of deterministic chaos, Geophys. Res. Lett., 17, 41-44, 1990.

Davis, T. N. and Sugiura, M., Auroral electrojet activity index AE and its universal time variation, J. Geophys. Res., 71 (3), 785801, 1966.

Ding, M., Grebogi, C., Ott, E., Sauer, T., and York, J. A., Estimating correlation dimension from a chaotic time series: when does a plateau onset occur? Physica D, 69, 404-424, 1993.

Elsner, J. B., and Tsonism, A. A., Singular spectrum analysis, a new tool in time series analysis, Plenum Press, New York, 1996.

Hones, E. W., Transient phenomena in the magnetotail and their relation to substorms, Space Sci. Rev., 23, 393-410, 1979.

Klimas, A. J., Baker, D. N., Roberts, D. A., Fairfield, D. H., and Buchner, J. A., A Nonlinear dynamic model of substorms, Geophys. Monog. Ser., vol. 64, edited by J.R. Kan, T.A. Potemra, S. Kokumun, and Iijima, pp. 449-459, AGU, Washington, D.C., 1991.

Klimas, A. J., Baker, D. N., Roberts, D. A., and Fairfield, D. H., A nonlinear dynamical analogue model of geomagnetic activity, J. Geophys. Res., 97, 12253-12266, 1992.

Klimas, A. J., Vassiliadis, D., Baker, D. N., and Roberts, D. A., The organized nonlinear dynamics of the magnetosphere, J. Geophys. Res., 101, 13089-13113, 1996.

Kugiumtzis D., State space reconstruction parameters in the analysis of chaotic time series-the role of the time window length, Physica D, Vol. 95, 13-28, 1996.

Liu, Z. X., Lee, L. C., Wei, C. Q., and Akasofu, S., I. Magnetospheric substorms: an equivalent circuit approach, J. Geophys. Res., 93, 7355-7366, 1988.

Osborne, A. R. and Provenzale, A., Finite correlation dimension for stochastic systems with power-low spectra, Physica D, 35, 357381, 1989.

Pavlos, G. P., Magnetospheric dynamics, in Proc. Symposium on Solar and Space Physics, edited by D. Dialetis, pp. 1-43, National Observatory of Athens, Athens, 1988.

Pavlos, G. P., Rigas, A. G., Dialetis, D., Sarris, E. T., Karakatsanis, L. P., and Tsonis, A. A., Evidence for chaotic dynamics in outer solar plasma and the earth magnetopause, in Chaotic Dynamics: Theory and Practice, edited by A. Bountis, pp. 327-339. Plenum, New York, 1992a.

Pavlos, G. P., Kyriakou, G. A., Rigas, A. G., Liatsis, P. I., Trochoutos, P. C., and Tsonis, A. A., Evidence for strange attractor structures in space plasmas, Ann. Geophys., 10, 309-322, 1992b.

Pavlos, G. P., Diamadidis, D., Adamopoulos, A., Rigas, A. G., Daglis. I. A., and Sarris, E. T., Chaos and magnetospheric dynamics, Nonlin. Proc. Geophys., 1, 124-135, 1994.

Pavlos, G. P., Athanasiu, M., Kugiumtzis, D., Hantzigeorgiu, N., Rigas A. G., and Sarris, E. T., Nonlinear analysis of Magnetospheric data, Part I. Geometric characteristics of the AE index time series and comparison with nonlinear surrogate data, Nonlin. Proc. Geophys., 6, 51-65, 1999a.

Pavlos, G. P., Kugiumtzis, D., Athanasiu M., Hantzigeorgiu, N., Diamantidis D., and Sarris, E. T., Nonlinear analysis of Magnetospheric data, Part II. Dynamical characteristics of the AE index time series and comparison with nonlinear surrogate data, Nonlin. Proc. Geophys., 6, 79-98, 1999b. 
Pavlos, G. P. Athanasiu M., Diamantidis D., Rigas A. G., and Sarris, E., Comments and new results about the magnetospheric chaos hypothesis, Nonlin. Proc. Geophys., 6, 99-127, 1999c.

Prichard, D. J, and Price, C. P., Spurious dimensions estimates from time series of geomegnetic indices, Geophys. Res. Lett., 19, 1623-1626, 1992.

Prichard, D. J. and Price, C. P., Is the AE Index re result of nonlinear dynamics? Geophys. Res. Lett., 20, 2817-2820, 1993.

Prichard, D. J., Short comment for magnmetospheric chaos, Nonlinear Proc. Geophys, 20,771-774, 1995.

Provenzale, A., Osborne, A. R., Kirwan, Jr. A. D., and Bergamasco, L., The study of fluid parcel trajectories in large-scale ocean flows, in Nonlinear Topics in Ocean Physics, edited by A.R. Osborne, pp. 367-402, Elsevier, Paris, 1991.

Provenzale, A., Smith, L. A., Vio, R., and Murante, G., Distinguishing low-dimensional dynamics and randomness in measured time series, Physica D, 58, 31-49, 1992.

Roberts, D. A., Baker, D. N., Klimas A. J., and Bargatze, L. F., Indications of low-dimensionality in magnetospheric dynamics, Geophys. Res. Lett., 18, 151-154, 1991.

Sarma, A. S., Vassiliadis D., and Papadopoulos, K., Reconstruction of low-dimensional dynamics by singular spectrum analysis, Geophys. Res. Lett., 20, 335-338, 1993.

Shan, H., Hansen, P., Goertz, C. K., and Smith, K. A., Chaotic appearance of the AE Index, Geophys. Res. Lett., 18, 147-150, 1991.

Shaw, R. Z., The dripping faucet as a model dynamical system, Aerial Press, Santa Cruz, CA, 1984.

Schreiber, T. and Schmitz A., Improved surrogate data for nonlin- earity test, Phys. Rev. Lett., 77, 635-638, 1996.

Takalo, J. and Timonen, J., Properties of AE data and bicolored noise, J. Geophys. Res., 99, 13239-13249, 1994.

Takens, F., Detecting strange attractors in turbulence, in Vol. 898 of Lectures Notes in Mathematics, edited by D.A. Rand and L.S. Young, pp. 366-381, Spinger, Berlin, 1981.

Takens, F., On the numerical determination of the dimension of an attractor, in dynamical systems and bifurcations, Groningen 1984, in vol. 1125 of Lecture Notes in Mathematics, pp. 99-106, edited by B.L.J. Braakama, H.W. Broer, and F. Takens, Berlin, Springer-Verlag, 1985.

Theiler, J., Some comments on the correlations dimensions of $1 / \mathrm{f} \mathrm{a}$ noise, Phys. Lett. A, 155, 480-493, 1991.

Theiler, J., Galdikian, B., Longtin, A., Eubank, S., and Farmer, J.D., Using surrogate data to detect nonlinearity in time series, in Nonlinear Modeling and Forecasting, vol. XII of SFI studies in the Sciences of Complexity, edited by M. Casdagli and S. Eubank, p. 163-188, Addison-Wesley, Reading, Mass., 1992a.

Theiler, J., Eubank, S., Longtin, A., Galdikian, B., and Farmer, J. D., Testing for nonlinearity in time series: the method of surrogate data, Physica D, 58, 77-94, 1992b.

Vassiliadis, D., Sharma, A. S., Eastman, T. E., and Papadopoulos, K., Low-Dimensional chaos in magnetospheric activity from AE time series, Geophys. Res. Lett. 17, 1841-1844, 1990.

Vassiliadis, D., Sharma, A. S., and Papadopoulos, K., Time series analysis of magnetospheric activity using nonlinear dynamical methods, in Chaotic Dynamics: Theory and Practice, edited by T. Bountis, Plenum, New York, 1992 